Article

\title{
Uncertainty Quantification of Non-Dimensional Parameters for a Film Cooling Configuration in Supersonic Conditions
}

\author{
Simone Salvadori ${ }^{1, *(\mathbb{D})}$, Mauro Carnevale ${ }^{2} \mathbb{( D}$, Alessia Fanciulli ${ }^{1}$ and Francesco Montomoli ${ }^{3}$ (I) \\ 1 Department of Industrial Engineering (DIEF), University of Florence, 50139 Florence, Italy \\ 2 Department of Mechanical Engineering, University of Bath, Bath BA2 7AY, UK \\ 3 Department of Aeronautics, Imperial College of London, London W12 0NN, UK \\ * Correspondence: simone.salvadori@unifi.it; Tel.: +39-055-275-8779
}

Received: 15 July 2019; Accepted: 7 August 2019; Published: 10 August 2019

\begin{abstract}
In transonic high-pressure turbine stages, oblique shocks originating from vane trailing edges impact the suction side of each adjacent vane. High-pressure vanes are cooled to tolerate the combustor exit-temperature levels, then it is highly probable that shock impingement will occur in proximity to a row of cooling holes. The presence of such a shock, together with the inevitable manufacturing deviations, alters the location of the shock impingement and of the performance parameters of each cooling hole. The present work provides a general description of the aero-thermal field that occurs on the rear suction side of a cooled vane. Computational Fluid Dynamics (CFD) is used to evaluate the deterministic response of the selected configurations in terms of adiabatic effectiveness, discharge coefficient, blowing ratio, density ratio, and momentum ratio. Turbulence is modelled by using both the Shear Stress Transport method (SST) and the Reynolds Stress Model (RSM) implemented in ANSYS ${ }^{\circledR}$ FLUENT ${ }^{\circledR}$. The obtained results are compared with the experimental data obtained by the Institut für Thermische Strömungsmaschinen in Karlsruhe. Two uncertainty quantification methodologies based on Hermite polynomials and Padè-Legendre approximants are used to consider the probability distribution of the geometrical parameters and to evaluate the response surfaces for the system response quantities. Trailing-edge and cooling-hole diameters have been considered to be aleatory unknowns. Uncertainty quantification analysis allows for the assessment of the mutual effects on global and local parameters of the cooling device. Obtained results demonstrate that most of the parameters are independent by the variation of the aleatory unknowns while the standard deviation of the blowing ratio associated with the hole diameter uncertainty is around $12 \%$, with no impact by the trailing-edge thickness. No relevant advantages are found using either SST model or RSM in combination with Hermite polynomials and Padè-Legendre approximants.
\end{abstract}

Keywords: uncertainty quantification; film cooling; shock waves; computational fluid dynamics; turbulence modelling

\section{Introduction}

A widely used strategy to increase efficiency and specific power output of gas turbines consists of increasing the Turbine Entry Temperature (TET). Therefore, metal temperatures approach the melting point of the alloys used in high-pressure nozzles, which are greatly loaded as shown in [1]. Advanced cooling systems such as film cooling are necessary to protect nozzles from high temperatures. Such technology consists of the injection of coolant flow spilled from the high-pressure compressor stage to create a protecting fluid film on the blade surface. The interaction of the coolant with the main 
flow increases complexity in the flow's structures. The insurgence of counter-rotating vortices (called "kidney-shaped vortices") with a velocity component perpendicular to the surface is described in [2]. These vortices are generated by the redistribution of the vorticity content in the boundary layer and play a key role in the performance of the cooling devices. Kidney vortices cause a lift-off effect moving the coolant away from the wall and reducing the capability of film cooling in covering and protecting the blade surface. These three-dimensional structures are numerically investigated over flat plates in [3]. They noticed that the kidney vortices are greatly influenced by the boundary layer development inside the coolant channel. An additional whirling structure, the so-called "tornado effect", is described in [4], where the presence of a core-wise, cross-flow transport in hairpin vortices (created by coolant injection) in the laminar boundary layer is shown. This core-wise phenomenon continuously moves the fluid from the wall to the free stream. Similar structures are described in [5] for a turbulent boundary layer and in [6] in presence of interaction between a shock and the boundary layer.

The complexity of the fluid structures is further increased when the film cooling device is considered in a realistic turbomachinery configuration. The effect of the cooling position on the losses of a turbine stage is analyzed in [7]. They highlighted that the position and the inclination of a cooling jet strongly affects both the losses and the capability of the boundary layer to resist to an adverse pressure gradient. Moreover, in high-pressure stages oblique shocks shed from the Trailing Edge (TE) impinge onto the suction side of the adjacent vane. In case of presence of a row of film cooling holes their effectiveness is affected by the three-dimensional interaction between the cooling flow, the boundary layer development, and the shock itself. Several authors demonstrate a non-negligible variation in the local value of adiabatic effectiveness $\eta_{a w}$ when the hole is located a few diameters before the shock impingement zone [8-10].

More recently Uncertainty Quantification (UQ) methodologies have been coupled with CFD simulations, providing the opportunity to quantify the stochastic variations associated with the inevitable manufacturing deviations and the fluctuation of boundary conditions in an engine-like environment [11]. The sensitivity of film cooling performance to relevant non-dimensional parameters is discussed in [12] without a statistical analysis. A UQ analysis is used in [13] to demonstrate that small variations in the coolant duct geometry affect the coolant flow redistribution in a non-negligible way. These kinds of deviations are generally known as aleatory uncertainties. The different capability to propagate aleatory uncertainties using different CFD approaches such as Reynolds-Averaged Navier-Stokes (RANS), unsteady RANS or Large Eddy Simulation (LES), provides identification on the accuracy of different numerical approach as shown by [14]. These deviations are known in open literature as epistemic uncertainty and are more related to the limitations of the numerical model. The quantification of the epistemic uncertainties is still an open problem.

In this work the effect of an impinging shock is studied numerically using a test case designed by the Institut für Thermische Ströemungsmaschinen in Karlsruhe $[9,15]$. A shaped plate positioned at the mid-height of a converging nozzle creates a diverging region where the Mach number reaches $M a=1.5$, while at the end of the plate the flow slows down through a shock system. Two geometrical parameters are perturbed to introduce the aleatory uncertainty associated with manufacturing deviations, namely the diameter of both the plate trailing edge $\left(\mathrm{D}_{T E}\right)$ and the cooling hole $\left(\mathrm{D}_{c}\right)$. The deterministic maps of adiabatic effectiveness $\eta_{\text {aw }}$ close to the hole exit section are obtained using two different turbulence models (the SST model [16] and the Reynolds Stress Model [17]) and compared with the available experimental data. The deterministic values and the stochastic distribution of the performance parameters (blowing ratio $B R$, density ratio $D R$, momentum ratio $M R$ and discharge coefficient $C_{D}$ ) are also calculated using two Probabilistic Collocation Methods (PCM) with Hermite polynomials and Padè-Legendre approximants. Present work underlines the limitations of turbulence modelling in the analysis of $\eta_{a w}$ maps and shows that the impact of manufacturing uncertainty is not relevant for the calculation of the performance parameters except for $B R$, to which is associated a standard deviation up to $13.5 \%$. 


\section{Test-Case Description}

Numerical simulations are widely used in cooperation with experimental tests, but only recently CFD simulations have been coupled with UQ to study a wide range of turbomachinery problems $[13,18,19]$. Concerning the present study, a complete description of the experimental facility is reported in [9] and a detailed numerical campaign using the $k-\omega$ turbulence model [20] has been reported in [10]. The proposed simulations have been carried out considering deterministic boundary conditions Figure 1 shows a sketch of the control volume that includes the converging nozzle, the central (shaped) plate, the cylindrical cooling hole, and the plenum. The main flow reaches sonic speed at the throat while the shape of the lower part of the contoured plate allows the flow to accelerate further to supersonic velocities. At the trailing edge, an oblique shock wave is generated, which impinges on the lower wall in a region immediately downstream of the cooling-hole exit position. The flow physics (including the effectiveness distribution obtained in [21]) is depicted in Figure 2, which is obtained for geometry and boundary conditions corresponding to the experiments. The coolant interacts with the main flow generating a weak shock, which merges with the oblique shock shed by the plate trailing edge that impinges on the lower wall. The $\eta_{a w}$ distribution is modified by the presence of the adverse pressure gradient that is responsible for a local growth of the boundary layer.

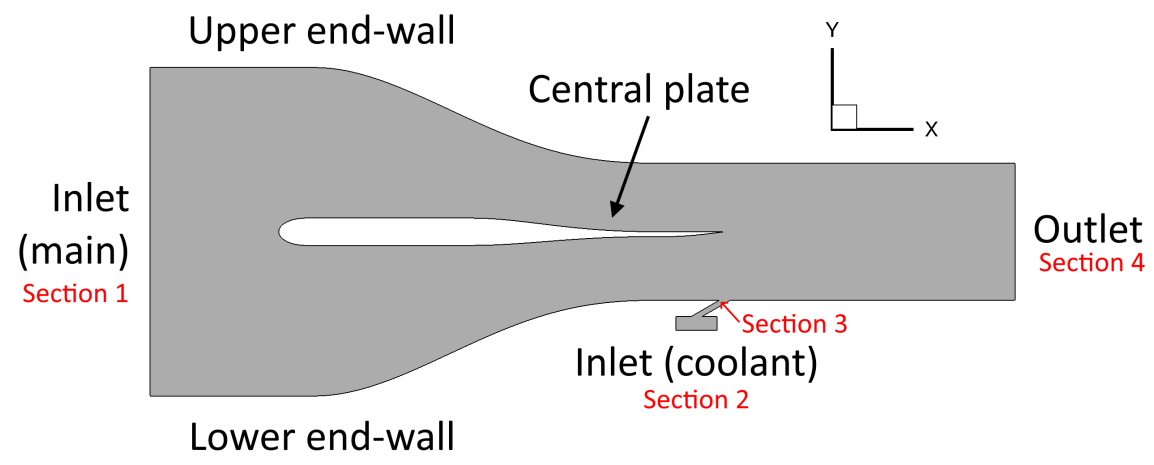

Figure 1. Control volume of the numerical simulation.

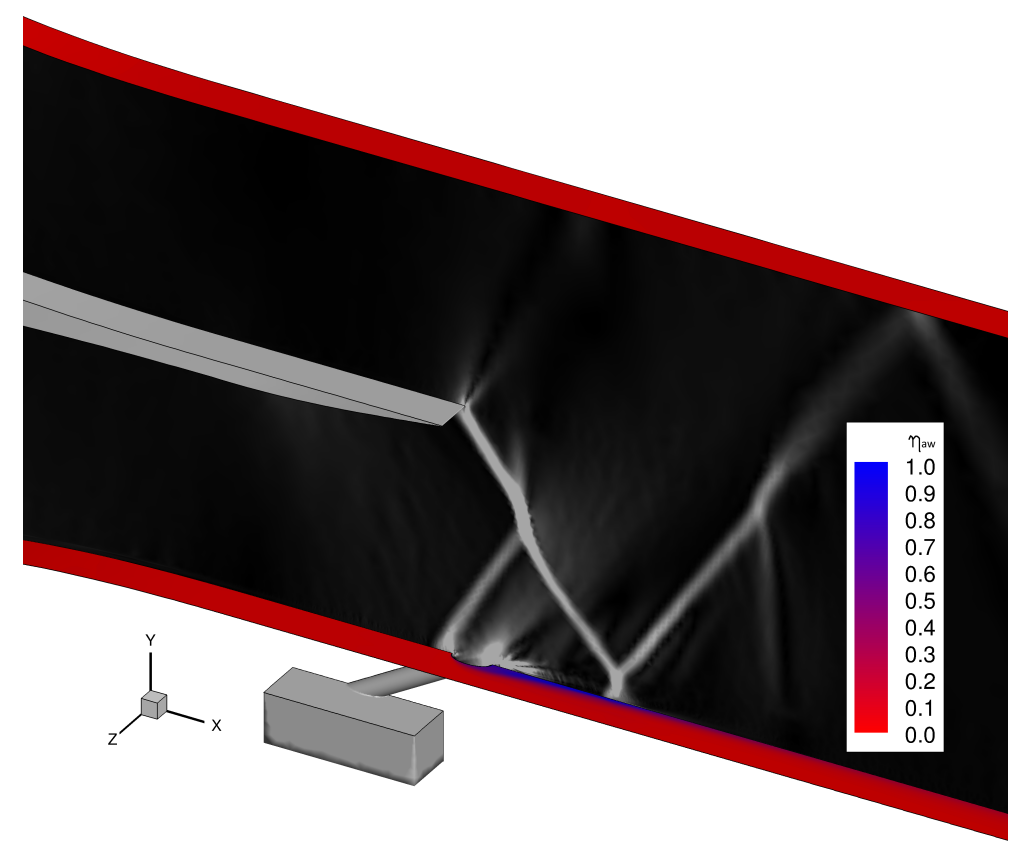

Figure 2. Main characteristics of the coolant/shock/boundary layer interaction for $M a=1.5$. 
In the experimental apparatus, five injection holes are located upstream of the shock impingement position and have a pitch-to-diameter ratio of 4.0 [9]. The control volume selected for the present numerical campaign includes only a portion of the entire domain, being the channel symmetric along the hole mid-plane and between two adjacent holes. Therefore, the computational domain is limited in the $\mathrm{z}$-direction by two symmetry planes that are set normal to the $\mathrm{x}-\mathrm{z}$ plane (see Figure 1 for reference). This choice neglects the formation of coolant oscillations on planes parallel to the $x-z$ plane and is coherent with the steady nature of the computations presented in the present work. The theoretical free stream Mach number at the shock location is around 1.5 and the blowing ratio value of 1.0 has been selected. The non-dimensional coolant total pressure is 1.01 with respect to main-flow inlet total pressure and the non-dimensional coolant total temperature is 0.557 with respect to main-flow inlet total temperature. For each investigated case, the numerical value has been calculated using Equation (1) in accordance with the experimental definition:

$$
B R=\frac{\rho_{c} \cdot u_{c}}{\rho_{m} \cdot u_{m}}
$$

In Equation (1) $\rho_{c}$ is the coolant density, $\rho_{m}$ is the main-flow density, $u_{c}$ is the coolant velocity and $u_{m}$ is the main-flow velocity. The value of the adiabatic effectiveness is essentially a non-dimensional representation of the adiabatic wall temperature $T_{a w}$ and is calculated using Equation (2):

$$
\eta_{a w}=\frac{T_{a w}-T_{r e c, m}}{T_{0 c}-T_{r e c, m}}
$$

where $T_{0 c}$ is the coolant stagnation temperature (evaluated approximately at the hole exit section) and $T_{r e c, m}$ is the recovery temperature of the main flow. In case of perfect coverage of the wall by the coolant flow $T_{a w}$ is equal to $T_{0 c}$ and $\eta_{a w}$ is 1.; in case of absence of coolant $T_{a w}$ is equivalent to $T_{r e c, m}$ and $\eta_{\text {aww }}$ is 0 .

Calculations with an adiabatic condition for the cooled plate are used to evaluate the adiabatic wall temperature distribution. The evaluation of the main-flow recovery temperature has been performed using Equation (3):

$$
T_{r e c, m}=T_{0 m} \cdot \frac{1+r \cdot \frac{\gamma-1}{2} M a_{i s}^{2}}{1+\frac{\gamma-1}{2} M a_{i s}^{2}}
$$

The value of the recovery temperature is a function of the recovery factor $r$ that in case of turbulent flows can be estimated as $r=\operatorname{Pr}^{0.33}$ [22].

\section{Numerical Methodology}

The computational domain assumes flow symmetry at the hole centerline and between two adjacent holes. That assumption is justified both by the fact that the boundary conditions are uniform and that unsteady effects in the lateral direction are expected to be negligible. As shown in Figure 1, the control volume includes the main-flow inlet, a coolant supply plenum, and the cylindrical cooling channel, the end-walls that define the nozzle, the central shaped plate, and the outlet. The presence of the plenum in film cooling simulations is crucial to correctly evaluate the aerodynamic losses and the development of secondary flows [23-26] and then it is included in the present computational control volume. Steady RANS equations are solved using a pressure-based approach. Pressure-velocity equations are coupled, and the calculation is fully second-order accurate. The Shear Stress Transport (SST) model [16] and Reynolds Stress Model (RSM) [17] already implemented in the commercial ANSYS ${ }^{\circledR}$ FLUENT $^{\circledR}$ code are used as turbulence closures to try to overcome the limitations of the standard k- $\omega$ model [20]. The main difference between the two selected turbulence closures consists of the calculation of the stress-tensor. RSM can evaluate the non-isotropic features of turbulence while SST model considers the turbulence as an isotropic quantity. The comparison of the two different approaches is aimed at assessing the role of the non-isotropic turbulence features due to 
the shock/coolant interaction in the evaluation of the distribution of adiabatic effectiveness. Inlet turbulence level is set at $5 \%$ while a turbulence length scale of $1 \mathrm{~mm}$ has been considered. Walls are set as adiabatic and viscous heating is considered to make the heat transfer evaluation accurate. Calculation is converged when all the residuals are constantly below $10^{-5}$ and the mass-flow error is below $1 \%$.

The uncertainty quantification analysis has been performed by considering two geometrical deviations as aleatory parameter: (i) the diameter of the cooling channel; (ii) the diameter of trailing edge of the central plate. Both deviations are considered to obtain a uniform distribution. The deviations related to the geometrical parameters have been assumed as $10 \%$ of the nominal value for the coolant diameter and $20 \%$ for the trailing-edge diameter [11]. Selected deviations are slightly higher than the realistic ones to obtain a wider response surface as result. The test matrix of the numerical campaign is summarized in Table 1 using non-dimensional $D$ values with respect to the nominal configuration.

Table 1. Test matrix (with trailing-edge and coolant non-dimensional diameters.

\begin{tabular}{cccl}
\hline Geometry & $\mathbf{D}_{\text {TE }}[-]$ & $\mathbf{D}_{c}[-]$ & \\
\hline No. 1 & 1.2 & 0.9 & \\
No. 2 & 1.2 & 1.0 & nominal $D_{c}$ \\
No. 3 & 1.2 & 1.1 & \\
No. 4 & 1.0 & 0.9 & nominal $D_{T E}$ \\
No. 5 & 1.0 & 1.0 & nominal configuration \\
No. 6 & 1.0 & 1.1 & nominal $D_{T E}$ \\
No. 7 & 0.8 & 0.9 & \\
No. 8 & 0.8 & 1.0 & nominal $D_{c}$ \\
No. 9 & 0.8 & 1.1 & \\
\hline
\end{tabular}

The meshes used for the nine calculations (necessary for the UQ analysis) are generated using the commercial hybrid grid generator Centaur ${ }^{\mathrm{TM}}$ by Centaursoft. The mesh is denser in the region where the shock-boundary layer interaction is expected (see Figure 3a) and the overall quality of the mesh is much higher than in [10]. The number of tetrahedral elements in the coolant hole is increased as shown in Figure $3 b$, with high resolution of the channel flow. The generated grids are particularly accurate in the near-wall region, where a value of $y^{+}$always below 0.3 is obtained. The final mesh used for the nominal case contains approximately $7.8 \cdot 10^{6}$ elements, which trebles the number of elements used for the analysis showed in [10] for the same case. The main reason for the large increase of elements occurring in the present work is the refinement of the portion of control volume where shock/coolant interaction is expected. To include a smooth transition of the elements' dimensions all over the domain, also the other regions have been refined. All the used meshes include a similar number of elements and are generated using the same rules for elements sizing. Since the simulations in [10] have been demonstrated to be grid independent, the ones of the present work are also considered to be reliable for the present analysis.

The aims of the present numerical campaign can be summarized in the following list:

- to correctly capture the characteristic flow features of the present test case;

- $\quad$ to compare the deterministic distributions of $\eta_{\text {aw }}$ obtained with different turbulence models;

- to propose a reliable strategy for the calculation of non-dimensional performance parameters;

- to compare the performance of two different polynomials bases in the evaluation of aleatory uncertainty;

- to quantify the uncertainty associated with manufacturing deviations for the calculation of non-dimensional performance parameters (except for $\eta_{a w}$ );

- to see how uncertainty propagates depending on the turbulence model. 


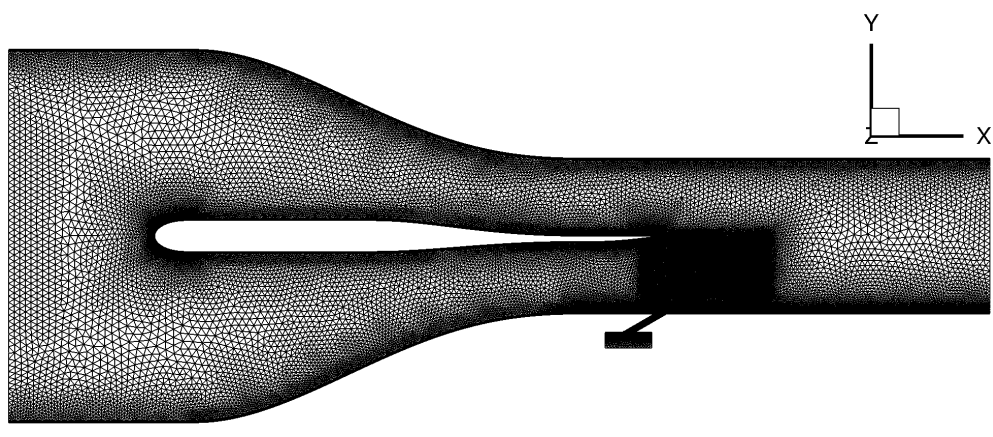

(a) Computational mesh of the control volume

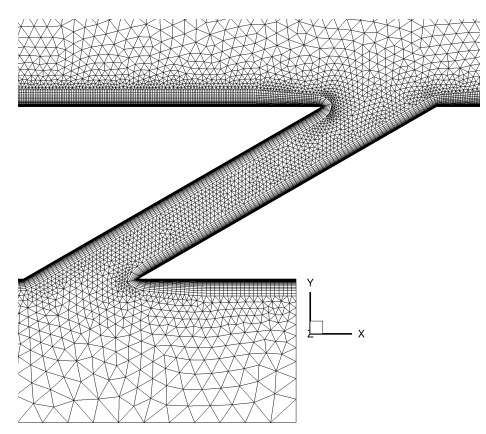

(b) Cooling-hole mesh

Figure 3. Computational mesh.

\section{Definition of the Non-Dimensional Parameters}

In this section, the non-dimensional parameters that will be calculated using the outcomes of the simulations are defined.

\subsection{Density Ratio}

The density ratio $D R$ is a non-dimensional parameter defined as the ratio between the coolant density $\rho_{c}$ and the main-flow density $\rho_{m}$ (Equation (4)):

$$
D R=\frac{\rho_{c}}{\rho_{m}}
$$

\subsection{Momentum Ratio}

The momentum ratio $M R$ is a non-dimensional parameter defined as the ratio between the coolant momentum $\rho_{c} u_{c}^{2}$ and the main-flow momentum $\rho_{m} u_{m}^{2}$ (Equation (5)):

$$
M R=\frac{\rho_{c} u_{c}^{2}}{\rho_{m} u_{m}^{2}}
$$

\subsection{Discharge Coefficient}

The discharge coefficient $C_{D}$ is a non-dimensional parameter that gives an estimation of the aerodynamic losses generated both by the local separation of the flow at the cooling channel inlet section and by the development of the boundary layer. It is defined analytically as the ratio between the mass-flow entering the cooling hole and the ideal mass-flow (Equation (6)). The latter is calculated considering an isentropic expansion between a plenum characterized by the coolant stagnation pressure and temperature values and a plenum where the pressure value is the one of the main flow at the outlet section of the hole. The passage area to be considered corresponds to the one at the entrance of the cooling hole.

$$
C_{D}=\frac{\dot{m}_{c}}{p_{0 c} \cdot\left(\frac{p_{m}}{p_{0 c}}\right)^{\frac{\gamma+1}{2 \gamma}} \cdot\left(\frac{2 \gamma}{(\gamma-1) \cdot R T_{0 c}} \cdot\left(\left(\frac{p_{0 c}}{p_{m}}\right)^{\frac{\gamma-1}{\gamma}}-1\right)\right)^{\frac{1}{2}} \cdot \frac{\pi D^{2}}{4}}
$$

where $\dot{m}_{c}$ is the coolant mass-flow, $p_{0 c}$ is the coolant stagnation pressure, $p_{m}$ is the main-flow static pressure value at the hole exit section, $T_{0 c}$ is the coolant stagnation temperature and $D$ is the diameter of the cooling-hole inlet section. 


\section{Data Processing}

To evaluate the performance of film cooling for the current configuration, the parameters defined in the previous section must be calculated using the deterministic results of the numerical simulations. Considering the control volume defined in Figure 1, it is possible to assign a specific number to each section:

- $\quad$ section 1 is the main-flow inlet;

- $\quad$ section 2 is the cooling-hole inlet section;

- $\quad$ section 3 is the cooling-hole outlet section;

- $\quad$ section 4 is the main-flow outlet section.

Among the necessary values for the calculation of the parameters, the ones in the following list are known:

- fluid properties:

gas constant $R=287\left[\mathrm{Jkg}^{-1} \mathrm{~K}^{-1}\right]$;

the ratio between the specific heats $\gamma=c_{p} / c_{v}=1.4[-]$;

- control volume geometrical characteristics:

main-flow inlet area $A_{\text {main }}\left[\mathrm{m}^{2}\right]$;

coolant inlet area $A_{\text {plenum }}\left[\mathrm{m}^{2}\right]$;

hole diameter $D[m]$;

coolant hole section area $A_{\text {hole }}\left[\mathrm{m}^{2}\right]$;

- fluid dynamics boundary conditions:

stagnation pressure $p_{01}[\mathrm{~Pa}]$ and stagnation temperature $T_{01}[\mathrm{~K}]$ at main-flow inlet section;

stagnation pressure $p_{02}[\mathrm{~Pa}]$ and stagnation temperature $T_{02}[\mathrm{~K}]$ at coolant inlet section;

- $\quad$ values obtained from each converged simulation:

coolant mass-flow $\dot{m}_{c}[\mathrm{~kg} / \mathrm{s}]$;

main-flow pressure calculated on the plate before the hole exit section $p_{m}[\mathrm{~Pa}]$;

coolant pressure calculated on the plate before the hole exit section $p_{c}[\mathrm{~Pa}]$.

The isentropic relations and the perfect gas law are used to complete the definition of the non-dimensional parameters [27]:

$$
\begin{aligned}
\frac{T_{0}}{T}=1 & +\frac{\gamma-1}{2} M a^{2} \\
\frac{p_{0}}{p}=\left(\frac{T_{0}}{T}\right)^{\gamma /(\gamma-1)} & =\left(1+\frac{\gamma-1}{2} M a^{2}\right)^{\gamma /(\gamma-1)} \\
\frac{\rho_{0}}{\rho}=\left(\frac{T_{0}}{T}\right)^{1 /(\gamma-1)} & =\left(1+\frac{\gamma-1}{2} M a^{2}\right)^{1 /(\gamma-1)} \\
p & =\rho \cdot R T
\end{aligned}
$$

where $T_{0}, p_{0}$ and $\rho_{0}$ are the stagnation values, $T, p$ and $\rho$ are the static values and $M a$ is the Mach number. More information can be found in the nomenclature section. The Equation (7) is exact for a steady, adiabatic process while Equations (8) and (9) are valid for a steady, isentropic process only. Under these hypotheses, the non-dimensional parameters can be calculated as follows. 


\subsection{Blowing Ratio}

The blowing ratio has been defined in Equation (1). The main-flow mass flux $\left(\rho_{m} u_{m}\right)$ is determined through Equation (11):

$$
\left(\rho_{m} u_{m}\right)=p_{m} M a_{i s, m} \sqrt{\frac{\gamma}{R T_{m}}}
$$

where the $T_{m}$ value is evaluated using Equation (12):

$$
T_{m}=T_{01}\left(1+\frac{\gamma-1}{2} M a_{i s, m}^{2}\right)^{-1}
$$

where $T_{01}$ is the stagnation temperature in section 1 . The isentropic Mach number $M a_{i s, m}$ is calculated using Equation (13):

$$
M a_{i s, m}=\sqrt{\frac{2}{\gamma-1}\left[\left(\frac{p_{01}}{p_{m}}\right)^{\frac{\gamma-1}{\gamma}}-1\right]}
$$

The coolant mass flux $\left(\rho_{\mathcal{c}} \mathcal{u}_{\mathcal{c}}\right)$ is determined through Equation (14):

$$
\rho_{c} u_{c}=\frac{\dot{m}_{c}}{A_{\text {hole }}}
$$

\subsection{Density Ratio}

The density ratio has been defined in Equation (4). The main-flow density value $\rho_{m}$ is calculated using Equation (15):

$$
\rho_{m}=\rho_{01}\left(1+\frac{\gamma-1}{2} M a_{i s, m}^{2}\right)^{-\frac{1}{\gamma-1}}
$$

where the $M a_{i s, m}$ is the one calculated using Equation (13). The value of the stagnation density $\rho_{01}$ is calculated using Equation (16):

$$
\rho_{01}=\frac{p_{01}}{R T_{01}}
$$

The coolant density value $\rho_{c}$ is calculated using Equation (17):

$$
\rho_{c}=\rho_{02}\left(1+\frac{\gamma-1}{2} M a_{i s, c}^{2}\right)^{-\frac{1}{\gamma-1}}
$$

where the value of the stagnation density $\rho_{02}$ is calculated using Equation (18):

$$
\rho_{02}=\frac{p_{02}}{R T_{02}}
$$

while the $M a_{i s, c}$ is the one calculated using Equation (19):

$$
M a_{i s, c}=\sqrt{\frac{2}{\gamma-1}\left[\left(\frac{p_{02}}{p_{c}}\right)^{\frac{\gamma-1}{\gamma}}-1\right]}
$$

\subsection{Momentum Ratio}

The momentum ratio is defined according to Equation (5), which can be rewritten as reported in Equation (20):

$$
M R=\frac{\rho_{c} u_{c}^{2}}{\rho_{m} u_{m}^{2}}
$$


The main-flow momentum $\left(\rho_{m} u_{m}^{2}\right)$ is calculated as reported in Equation (21):

$$
\rho_{m} u_{m}^{2}=\gamma p_{m} M a_{i s, m}^{2}
$$

where the $M a_{i s, m}$ value is calculated using Equation (13). The coolant momentum $\left(\rho_{c} u_{c}^{2}\right)$ is calculated as reported in Equation (22):

$$
\rho_{c} u_{c}^{2}=\gamma p_{c} M a_{i s, c}^{2}
$$

where the $M a_{i s, c}$ value is calculated using Equation (19). Since both the main-flow and the coolant are modelled using air as perfect gas, they have the same value for $\gamma$. Therefore, considering Equations (21) and (22) the momentum ratio can be rewritten as in Equation (23):

$$
M R=\frac{p_{c} M a_{i s, c}^{2}}{p_{m} M a_{i s, m}^{2}}
$$

\subsection{Discharge Coefficient}

All the necessary values for the calculation of the discharge coefficient as defined in Equation (6) has been defined in the previous sections. Then, it is sufficient to substitute $p_{0 c}$ with $p_{02}$ to obtain Equation (24):

$$
C_{D}=\frac{\dot{m}_{\mathcal{C}}}{p_{02} \cdot\left(\frac{p_{m}}{p_{02}}\right)^{\frac{\gamma+1}{2 \gamma}} \cdot\left(\frac{2 \gamma}{(\gamma-1) \cdot R T_{02}} \cdot\left(\left(\frac{p_{02}}{p_{m}}\right)^{\frac{\gamma-1}{\gamma}}-1\right)\right)^{\frac{1}{2}} \cdot \frac{\pi D^{2}}{4}}
$$

\section{Discussion on the Deterministic Results}

In this section, numerical results are discussed in comparison with the available experimental data. The aim is to evaluate the overall accuracy of deterministic computations and the impact of turbulence modelling. The expected outcome is to capture both the most relevant flow features and the correct level of the non-dimensional performance parameters to guarantee a reliable uncertainty quantification analysis.

\subsection{Adiabatic Effectiveness}

Figure 4 shows the comparison between the numerical and experimental maps of adiabatic effectiveness as defined in Equation (2). As can be observed, the lateral mixing of the cooling flow is underestimated by the CFD simulations with respect to the experiments. Film cooling effectiveness is experimentally evaluated within an accuracy of $5 \%$ [28], which is not sufficient to justify the differences showed in Figure 4. The coolant is confined close to the centerline while the experiments show a wider (and non-symmetric) redistribution of the cooling flow. That kind of outcome is associated with the under-prediction of the vertical mixing in the wake of the jet for high $(\geq 1.0) B R$ values [29].

The agreement between the experimental and the numerical data in the centerline is demonstrated in [30] for the SST model and in [21] for RSM. In both the papers, a lift-off mechanism generated by the adverse pressure gradient caused by the incoming oblique shock (see Figure 2) is described that reduces the local $\eta_{a w}$ value. It can be also observed that the result obtained using the SST model shows a non-negligible impact of shock impingement at $x / D \approx 5.0$. That outcome can be ascribed to the isotropic nature of the SST model, while RSM is able to model the lateral mixing of the cooling flow thus reducing the effect of the shock. In addition, it can be observed that more accurate results are shown in [10] for lower $B R$ values, obtained using the baseline $\mathrm{k}-\omega$ model [20] with a different solver (the in-house HybFlow solver). The same outcomes are also shown in [29], where modifications to the turbulence model have no effects in improving predictions of wall temperatures in the wake region of 
detached films and the standard $\mathrm{k}-\omega$ model is sufficient to reproduce the experimental maps for low and moderate $B R$ values.

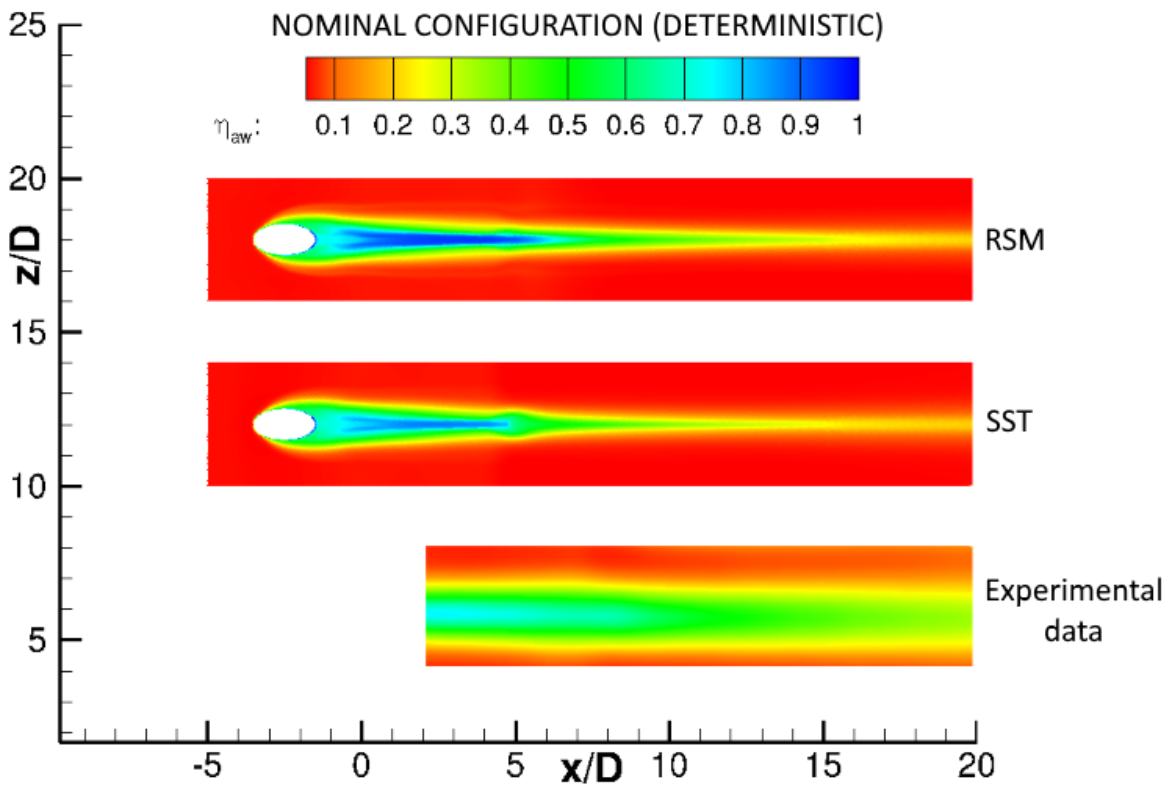

Figure 4. $\eta_{a w}$ experimental and numerical 2-D maps [21,30].

\subsection{Non-Dimensional Parameters}

The deterministic values of the non-dimensional performance parameters are reported in Tables 2 and 3 for the "Geom. 5" (nominal) configuration. As can be observed, the BR value is around 1.0, which is also the experimental value (within an accuracy of $6.2 \%$ [28]). Concerning the $D R$ and the $M R$ values, there are no experimental confirmations but their levels (respectively around 2.0 and 0.9) are coherent with the typical values found for the present configuration. The deterministic $C_{D}$ value is around 0.73 for most of the investigated configuration. It can be compared with the available experimental data (within an accuracy of 6.5\% [28]), with the numerical data obtained in [10] and with data showed for a similar configuration (at the lower Mach number of 1.2) in [31]. As can be observed in Figure $5, C_{D}$ values are coherent with the experimental data (black line with triangular symbols) and are much closer to them with respect to what is shown in [10] (red line with square symbols). The $C_{D}$ values obtained in the present work are also in line with the data shown in [31] for $M a=1.2$ (reported using light blue line with circular symbols with comparison purposes). In fact, although in [31] there are no data for $M a=1.5$ it is shown that increasing the main-flow Mach number the $C_{D}$ values decrease, thus moving toward the values found in the present case.

Table 2. Non-dimensional parameters for the film cooling configuration studied using the SST turbulence model.

\begin{tabular}{rccccccccc}
\hline SST Model & Geom. 1 & Geom. 2 & Geom. 3 & Geom. 4 & Geom. 5 & Geom. 6 & Geom. 7 & Geom. 8 & Geom. 9 \\
\hline$C_{D}[-]$ & 0.744 & 0.739 & 0.740 & 0.729 & 0.736 & 0.733 & 0.746 & 0.746 & 0.732 \\
$B R[-]$ & 0.811 & 0.995 & 1.204 & 0.794 & 0.990 & 1.194 & 0.813 & 1.004 & 1.192 \\
$D R[-]$ & 1.977 & 1.998 & 2.020 & 1.991 & 2.015 & 2.016 & 1.990 & 1.983 & 2.008 \\
$M R[-]$ & 0.921 & 0.910 & 0.897 & 0.908 & 0.898 & 0.893 & 0.914 & 0.917 & 0.898 \\
\hline
\end{tabular}


Table 3. Non-dimensional parameters for the film cooling configuration studied using the Reynolds Stress turbulence model.

\begin{tabular}{rccccccccc}
\hline RSM Model & Geom. 1 & Geom. 2 & Geom. 3 & Geom. 4 & Geom. 5 & Geom. 6 & Geom. 7 & Geom. 8 & Geom. 9 \\
\hline$C_{D}[-]$ & 0.746 & 0.736 & 0.737 & 0.733 & 0.732 & 0.731 & 0.741 & 0.740 & 0.729 \\
$B R[-]$ & 0.836 & 1.056 & 1.199 & 0.799 & 0.985 & 1.190 & 0.808 & 0.995 & 1.186 \\
$D R[-]$ & 1.987 & 2.002 & 2.018 & 1.991 & 2.020 & 2.010 & 1.984 & 1.992 & 2.010 \\
$M R[-]$ & 0.916 & 0.908 & 0.899 & 0.909 & 0.901 & 0.897 & 0.917 & 0.911 & 0.897 \\
\hline
\end{tabular}

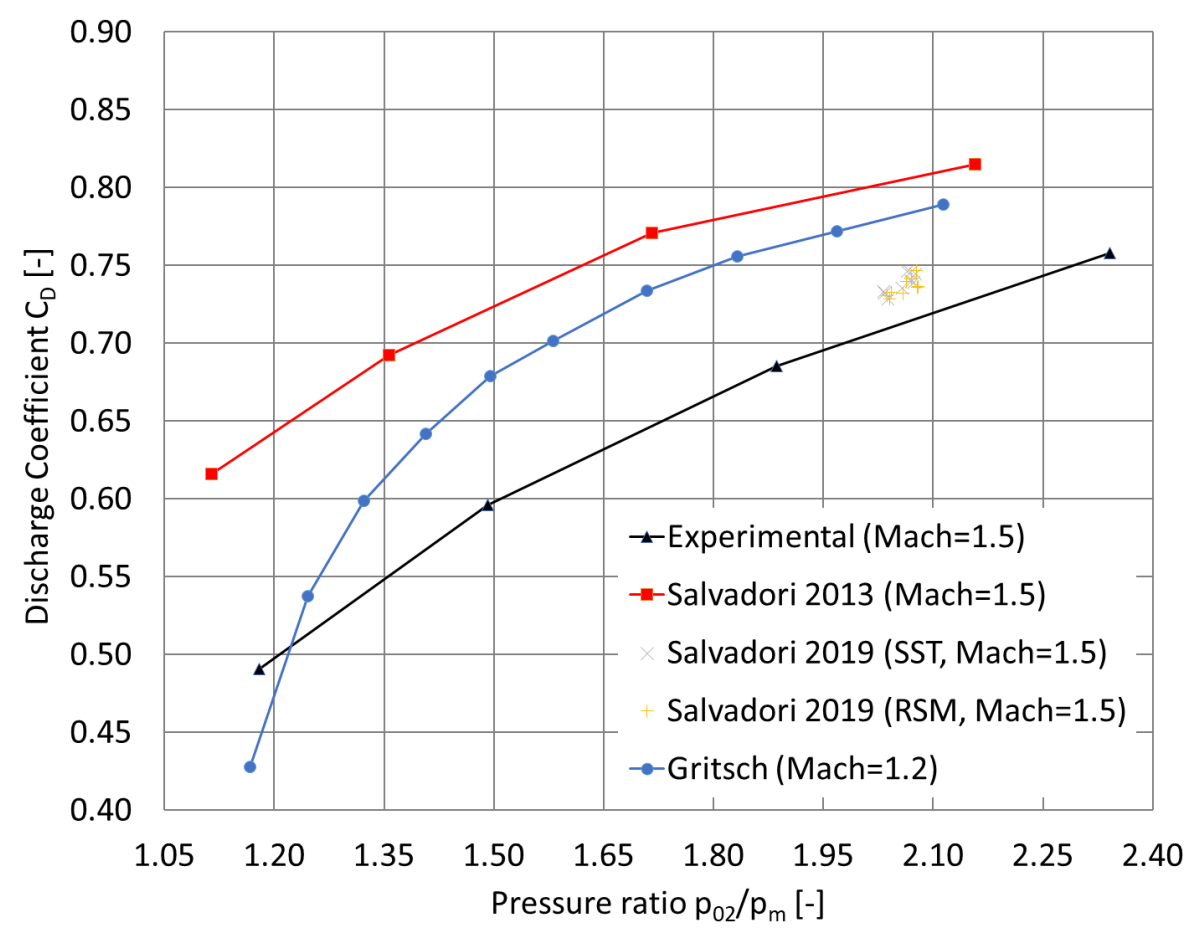

Figure 5. $C_{D}$ values obtained using the SST model and RSM $[10,31]$.

\subsection{Final Comments on the Deterministic Data}

The deterministic analysis demonstrated limited accuracy in the prediction of the 2-D maps of $\eta_{\text {aw }}$. However, the main features of the test case are captured and the calculation of the non-dimensional parameters (which is also the objective of the UQ analysis) gave good results when compared with the experimental data, being most of the values within the experimental accuracy. Furthermore, all the obtained results are coherent with what is found in recent literature [29]. A more accurate prediction of the $\eta_{\text {aw }}$ maps could be possible if one of the following approaches was used: (i) an unsteady computation; (ii) a hi-fidelity turbulence model, such LES.

The simulation of the unsteady interaction between the coolant and the main-flow would allow for the estimation of the enhanced mixing generated in the wake of the jets at the integral scales of turbulence by main-flow fluctuations (especially at higher $B R$ values). Furthermore, an unsteady computation would reproduce the oblique shock fluctuation generated by the vortex shedding that occurs at the TE of the central plate (similarly to what is shown in [32]). It must be underlined that the correct intensity of a shock can be correctly captured only by an unsteady analysis due to non-linear, unsteady stress terms [33].

Concerning the second point in the list, hi-fidelity CFD would correctly reproduce turbulence behavior, which is the driving mechanism for the mixing between the coolant and the main-flow in the inertial sub-range [25]. Unfortunately, the use of LES for the present test case is impracticable due to the high Mach number that would increase the computational time to a non-acceptable level for a UQ analysis. A similar comment can be done for unsteady computations, whose impact on the computational resources is not as relevant as using LES but is still not acceptable in the present 
activity, whose main aim is to analyze the impact of manufacturing uncertainty on the non-dimensional performance parameters. Being the latter well reproduced (at least within the experimental accuracy), the current numerical approach is used for the UQ analysis, while the improved reproduction of the $\eta_{\text {aw }}$ maps for the present case will be elaborated on in a separate numerical campaign.

\section{Uncertainty Quantification Methods for CFD}

The effect of manufacturing deviations on film cooling performance in presence of shocks has been studied by means of a non-intrusive statistical analysis. The proposed approach is based on the assumption that the perturbation of either boundary conditions or geometric features propagate linearly in the solution. Deviations in the prediction of a System Response Quantity (SRQ) depends on: (i) a Probability Distribution Function (PDF) that describes the deviation of the aleatory parameter; (ii) the adopted turbulence closure which defines the epistemic uncertainty; (iii) the physics involved in the flow structures. The statistical evaluation of the selected SRQ is obtained by performing a set of deterministic simulations with different boundary conditions, which are representative of the PDF of the aleatory uncertainty.

The efficiency and the reliability of the UQ approach depends on the adopted sampling technique. In the context of uncertainty quantification used along numerical simulations, the sampling process consists of collecting a set of results of deterministic analyses obtained for different boundary conditions. That sampling could be obtained using Monte Carlo approach, which implies many simulations [34]. As a consequence of the high computational demand, Monte Carlo procedure is not reliable when simulations are obtained using Computational Fluid Dynamics (CFD). A more suitable approach is based on the polynomial description of the stochastic variation. That approach allows for the determination of the PDF of the selected aleatory parameters by performing simulations only for a relatively small amount of boundary conditions. The investigated working points are obtained using the PCM procedure based on the representation of uncertainties on a linear space where the orthonormal basis is formed by a set of polynomials. According to this assumption, the propagation of an uncertainty parameter $\vec{x}$ associated with a PDF $\vec{\xi}$ within a physical system can be described in a polynomial form such as in Equation (25):

$$
y(\vec{x}, \vec{\xi})=\sum_{i=0}^{\infty} a_{i}(\vec{x}) \hat{u}(\vec{\xi})
$$

In Equation (25) $a_{i}$ and $\vec{x}$ are deterministic functions and $\hat{u}$ is a set of multidimensional orthogonal polynomials in the random variables $\xi$. Such methodology is usually known as Polynomial Chaos (PC) [35,36]. An extensive review of the methods is detailed in [37].

\subsection{Hermite Polynomials}

When aleatory uncertainty is associated with a normal distribution and the flow field is not affected by singularities, the basis $\hat{u}(\xi)$ on which the space of solutions can be reconstructed is formed by Hermite polynomials. The generic expansion of the stochastic response in Equation (25) can be expressed as reported in Equation (26):

$$
y(\vec{x}, \vec{\xi})=a_{0} H_{0}+a_{1} H_{1}+a_{2} H_{2}
$$

In a 1-D space, the form of the Hermite polynomials is reported in Equation (27) up to the second order, where $P_{n}$ is the $n^{\text {th }}$ order polynomial. A detailed description of a stochastic analysis based on the use of Hermite's polynomials for the study of aleatory uncertainty in turbomachinery-relevant problems is reported in [14]. In that work the inlet flow velocity in an internal cooling system is assumed as aleatory uncertainty. The inlet velocity is associated with a normal PDF and the boundary conditions are selected according a PCM based on Hermite's polynomials. Results demonstrated a 
correlation between aleatory and epistemic uncertainty in presence of non-linear problems dominated by turbulence. That kind of approach is the first one selected for the present activity.

$$
\begin{array}{ll}
\text { Order 0: } & P_{0}=H_{0}=1 \\
\text { Order 1: } & P_{1}=H_{1}=\xi_{1} \\
\text { Order 2: } & P_{2}=H_{2}=\xi_{1}^{2}-1
\end{array}
$$

\subsection{Padè-Legendre Approximants}

A different choice of polynomial basis is needed when the flow structure is characterized by discontinuities such as shocks, which is the case described in [6]. Shock-dominated flow structures can be modelled by Padè approximation as shown in [38]. The Padè approximation is a generalization of PC where a discontinuous response surface is described by a ratio of PC expansions. As this expansion can have poles, it can describe discontinuities. Numerator and denominator of the rational function are determined through a finite sum of orthogonal polynomials bases whose coefficients can be calculated from the function's values for a predefined set of points. Examples of this include Padè-Jacobi, Padè-Chebischev and Padè-Legendre (PL) approximants. The latter represent the second approach used in the present work following the prescriptions found in [38,39].

A complete basis of Legendre polynomials is defined by Equation (28):

$$
\left\langle P_{n}, P_{m}\right\rangle=\frac{1}{n+1 / 2} \delta_{n, m} \quad \text { with } \quad n, m \in N
$$

Polynomials are defined uniquely as $\left(1, x, \frac{1}{2}\left(3 x^{2}-1\right), \ldots\right)$ and the expansion can be continued to any desired order. Every function $u$ can be defined as a linear combination of Legendre's basis (Equation (29)):

$$
u=\sum_{n}^{\infty} \hat{u}_{n} P_{n}
$$

The coefficients $\hat{u}_{n}$ in the $n^{\text {th }}$ Legendre coefficient of $u$ are defined as in Equation (30):

$$
\hat{u}=\frac{\left\langle u, P_{n}\right\rangle}{\left\langle P_{u}, P_{n}\right\rangle}
$$

The function is known in specific discrete points and the Gaussian quadrature provides the discrete formulation where each scalar product in Equation (30) is defined as in Equation (31):

$$
\left\langle u, P_{n}\right\rangle_{N}=\sum_{j=0}^{N} P_{n}\left(x_{j}\right) P_{m}\left(x_{j}\right) \omega_{j} \quad \text { with } \quad \omega_{j}=\frac{2}{N(N+1)\left[P_{N}\left(x_{j}\right)\right]^{2}}
$$

The nodes $x_{i}$ in Equation (31) are the quadrature points and $\omega_{i}$ are the quadrature weights of the Gauss-Lobatto quadrature rule as described in [40]. The nodes are given as the roots of $P_{N+1}(x)$. Consequently, the nodes are chosen as the roots of the polynomial $P_{N}(x)$. The Gauss-Lobatto quadrature rule is chosen because it requires less function evaluations than standard Gauss quadrature. Given two integers $M$ and $L$, a Padè-Legendre approximation of a function $u$ is the ratio of two approximating polynomials $P_{M}$ and $Q_{L}$ based on the Legendre basis. The overall order of the reconstruction is $N=(M+L+1)$. Once the polynomial representation of the underlying function is obtained, it is possible to obtain mean $\hat{u}$ and variance $\hat{\sigma}$ directly from the functions in Equations (32) and (33):

$$
\begin{gathered}
\tilde{u}=\int_{D_{r n d}} u\left(x, \omega_{r n d}\right) f_{\omega} d \omega_{r n d} \\
\tilde{\sigma}=\int_{D_{r n d}} u\left(x, \omega_{r n d}\right)^{2} f_{\omega} d \omega_{r n d}-\left[\int_{D_{r n d}} u\left(x, \omega_{r n d}\right) f_{\omega} d \omega_{r n d}\right]^{2}
\end{gathered}
$$


In Equation (32) $\omega_{r n d}$ is the random parameter associated with the $\operatorname{PDF} f_{\omega}, D_{r n d}$ represents the domain of the random parameters and $x$ is the physical space.

\section{Discussion on the Statistical Analysis}

The aim of this section is to evaluate how manufacturing deviations affect non-dimensional parameters of a cooling device in supersonic conditions depending on turbulence modelling and UQ methodology. Deterministic values of $C_{D}, B R, D R$ and $M R$ are reported in Tables 2 and 3. Response surfaces for the parameters are visible from Figures 6-13. Finally, their mean values and standard deviations are reported in Table 4.

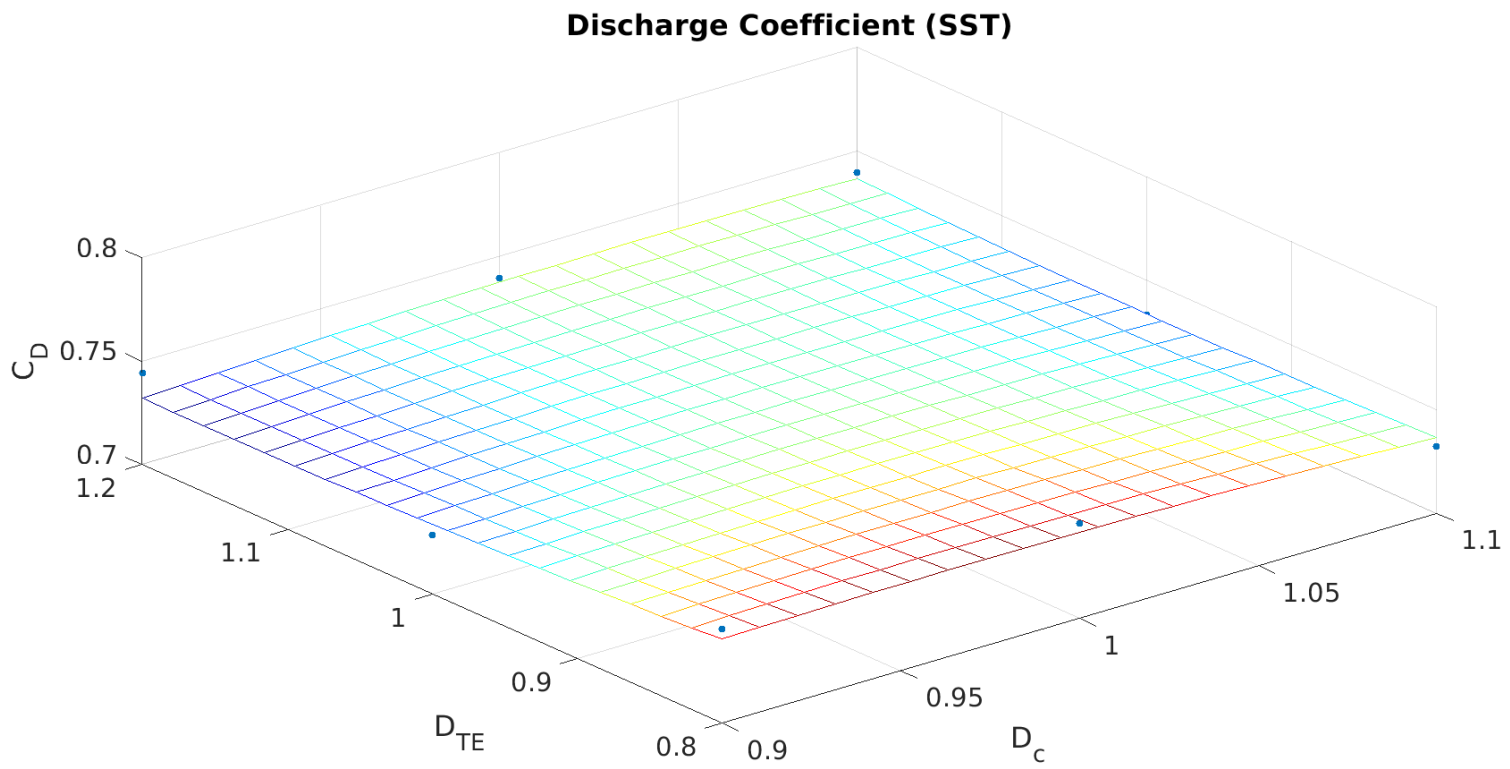

(a) SST model

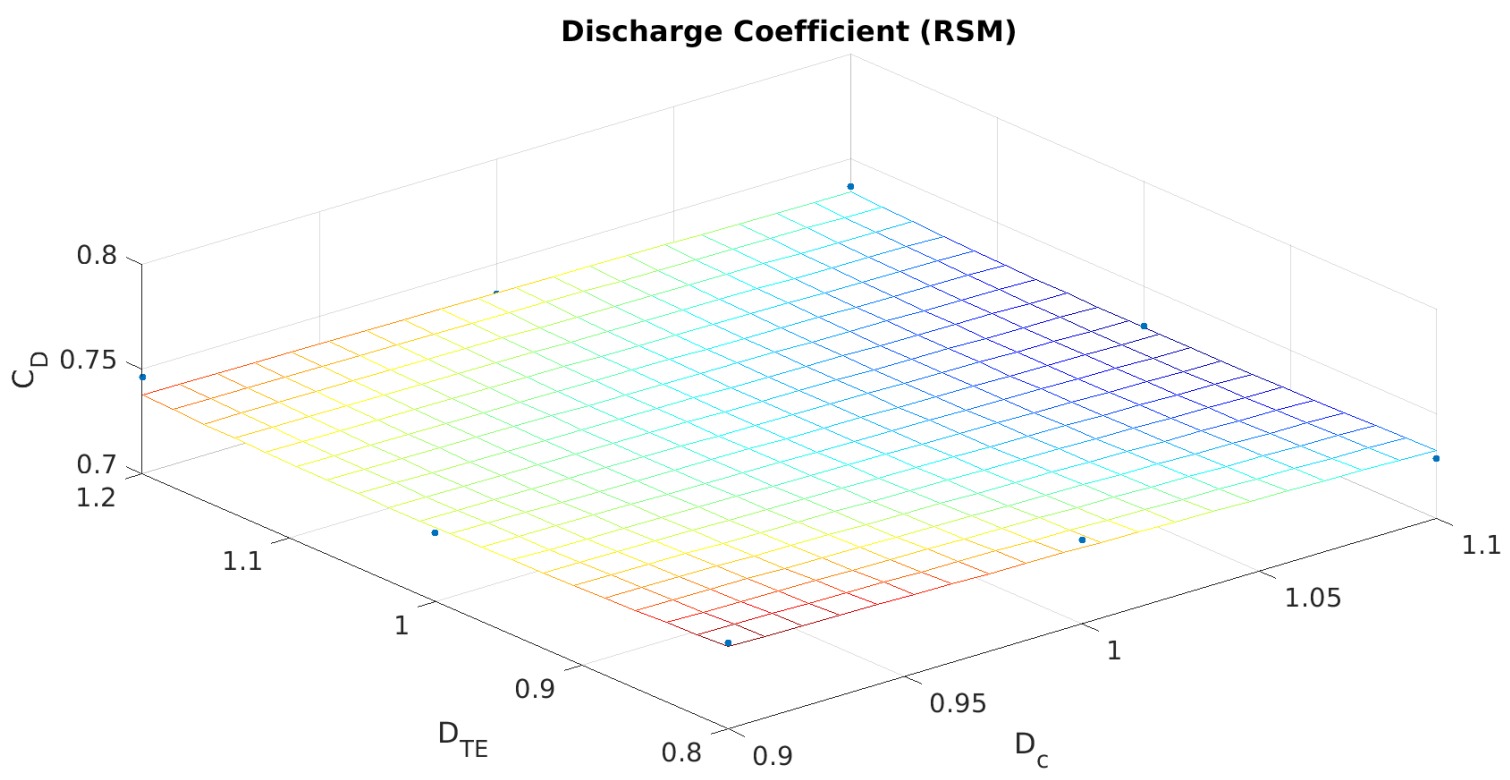

(b) Reynolds stress model

Figure 6. Discharge Coefficient $C_{D}$ (Hermite polynomials). 
Data included in Tables 2 and 3 show the dependence of the parameters on both the cooling-hole diameter and the plate's trailing-edge dimension. The $C_{D}$ value is almost constant (between 0.729 and 0.746) for the investigated configurations, which is an expected result. In fact, considering the definition of $C_{D}$ in Equation (24) and increasing the coolant channel diameter $\mathrm{D}_{c}$ both the actual and the isentropic mass-flows increase by similar amounts, with a limited impact by the variation of thermodynamic losses. Concerning the other parameters, $B R$ shows a variation up to $20 \%$ with respect to the nominal value (which is the one obtained for the nominal configuration, Geom. 5) and $D R$ and $M R$ are characterized by variations in the order of $1 \%$, irrespective of the selected turbulence model.

\section{Discharge Coefficient (SST)}

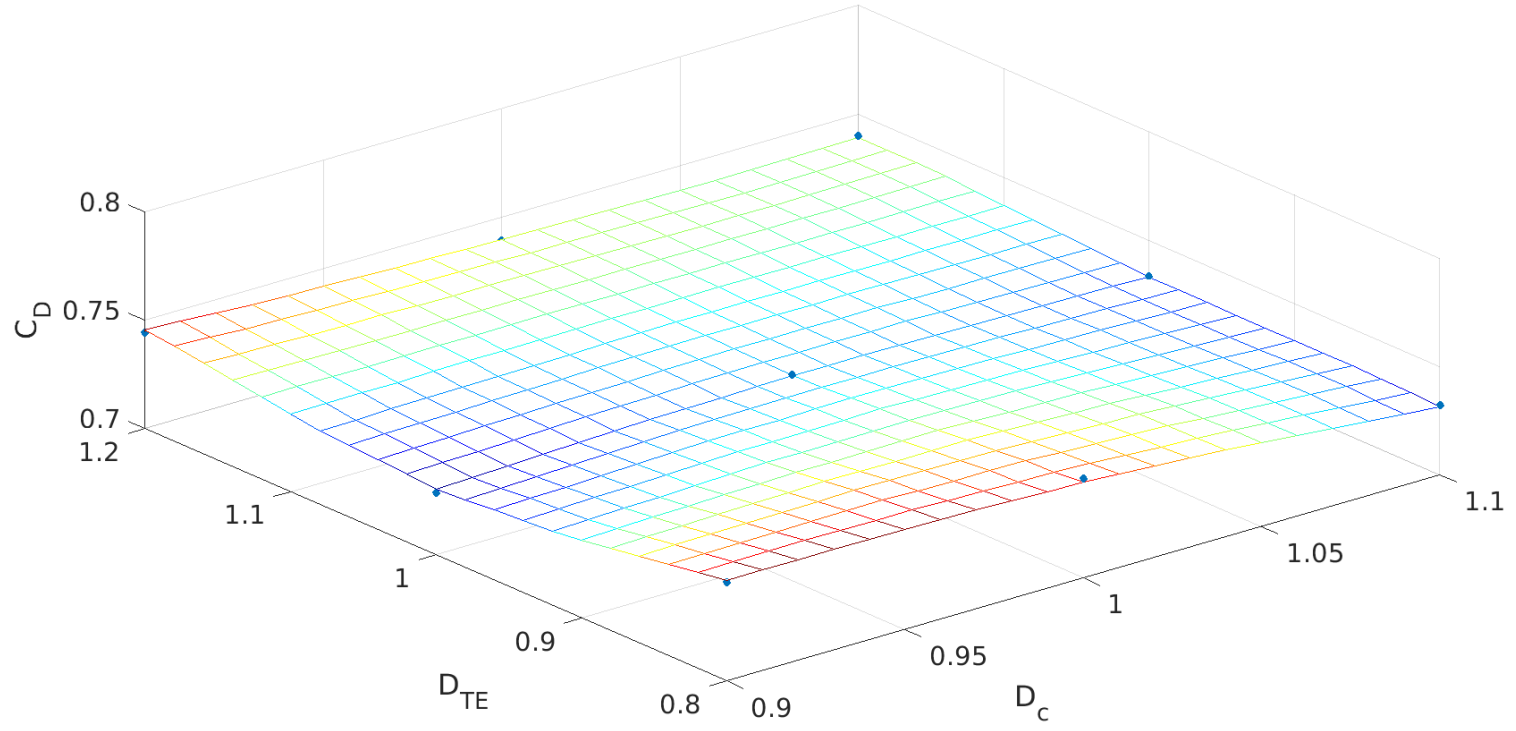

(a) SST model

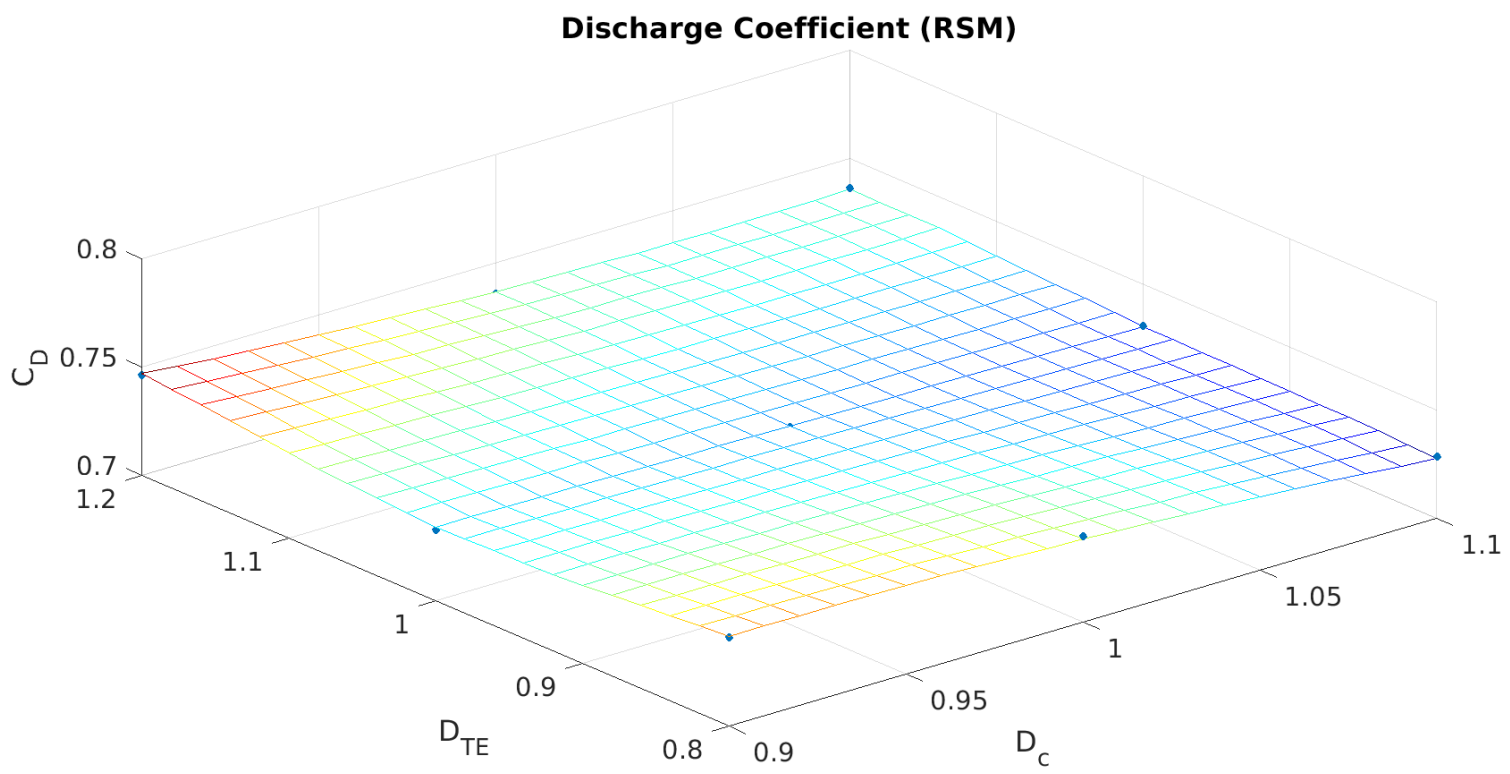

(b) Reynolds stress model

Figure 7. Discharge Coefficient $C_{D}$ (Padè polynomials). 
A detailed analysis of the obtained results can be performed looking at the Response Surfaces (RSs) showed from Figures 6-13. These RSs are obtained using both Hermite and Padè-Legendre polynomials to analyze the data showed in Tables 2 and 3.

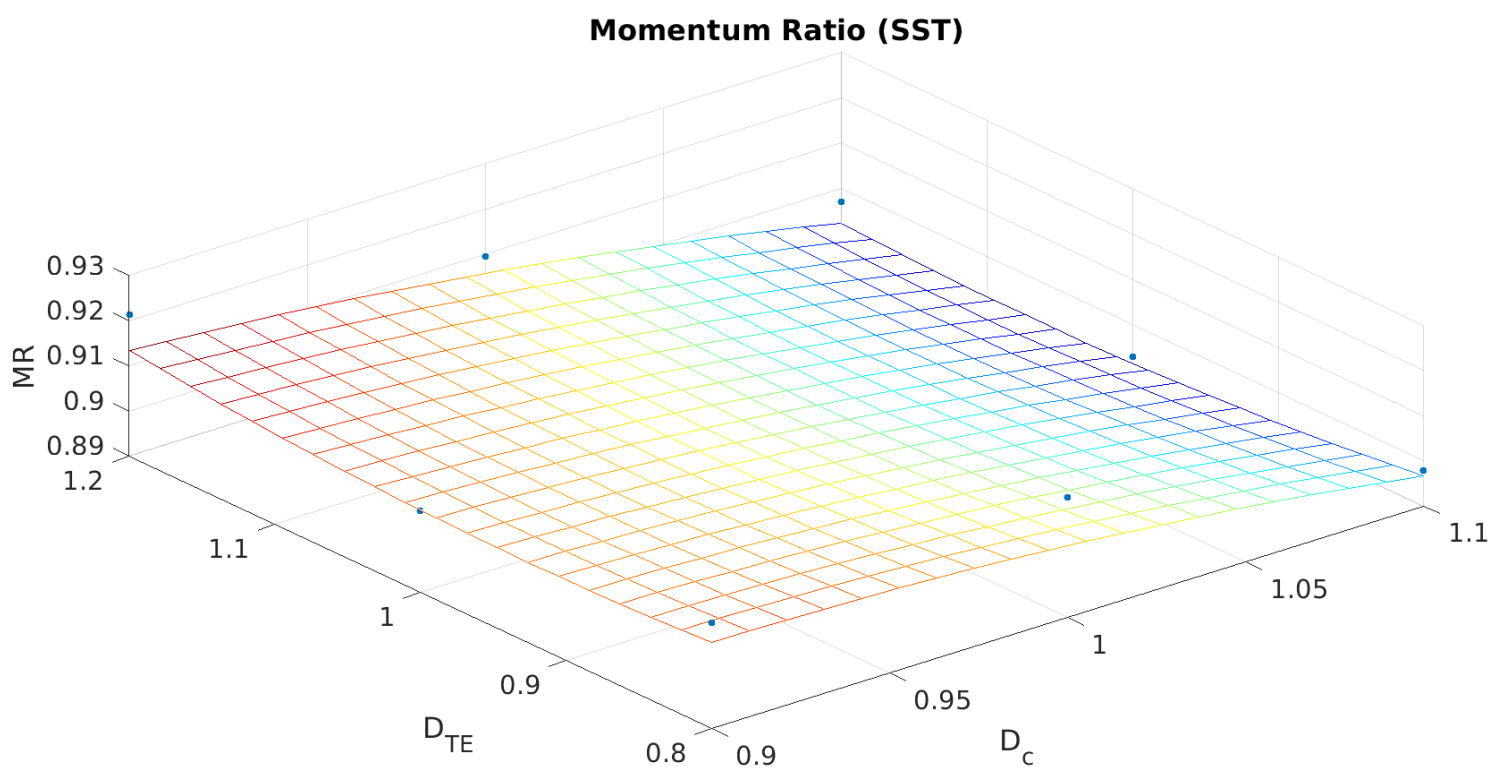

(a) SST model

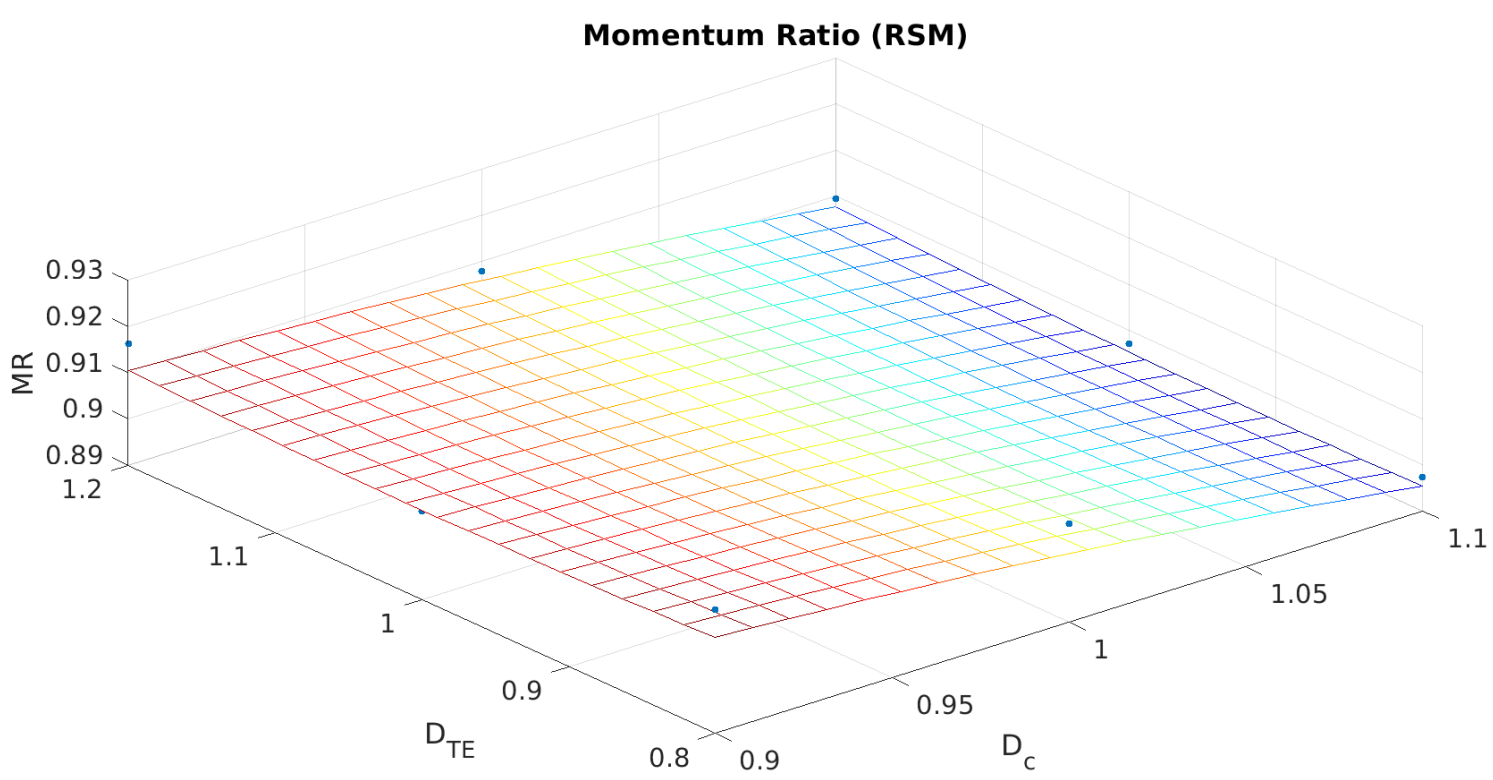

(b) Reynolds stress model

Figure 8. Momentum Ratio $M R$ (Hermite polynomials).

The outcome obtained using the $C_{D}$ values is showed from Figures $6 \mathrm{a}$ to $7 \mathrm{~b}$. As expected, the RSs are almost planar for all the investigated cases and there are limited differences between the SST and the RSM data. It can be observed that the selected approach based on the Hermite polynomials is limited to a second-order accuracy when applied to a set of 9 deterministic values, then the generation of a RS that could match all the deterministic values is not possible (see as example Figure 6). On the contrary, Padè-Legendre approximants can generate discontinuous RSs at sixth order with the same set of values, which gives the outcomes showed in Figure 7. The latter RSs accurately follows the 
deterministic values thanks to the superior ability to change the surface curvature. The same outcome can be observed by comparing Figures 9 and 11 with Figures 8 and 10 respectively. The ability of Padè-Legendre approximants in the generation of a RS that almost matches all the deterministic values obtained for $M R$ and $D R$ is guaranteed by the possibility to generate surfaces with quick curvature variations.

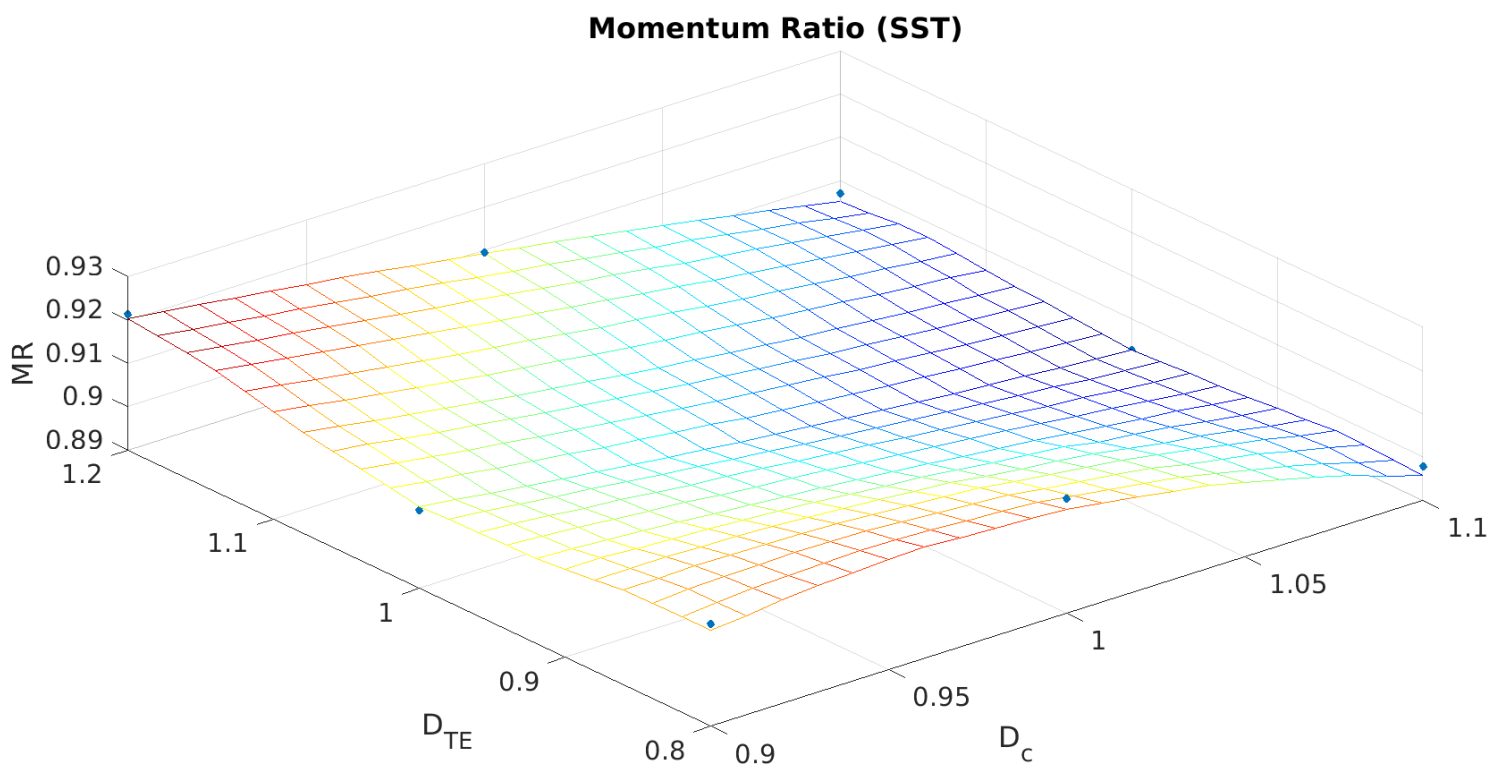

(a) SST model

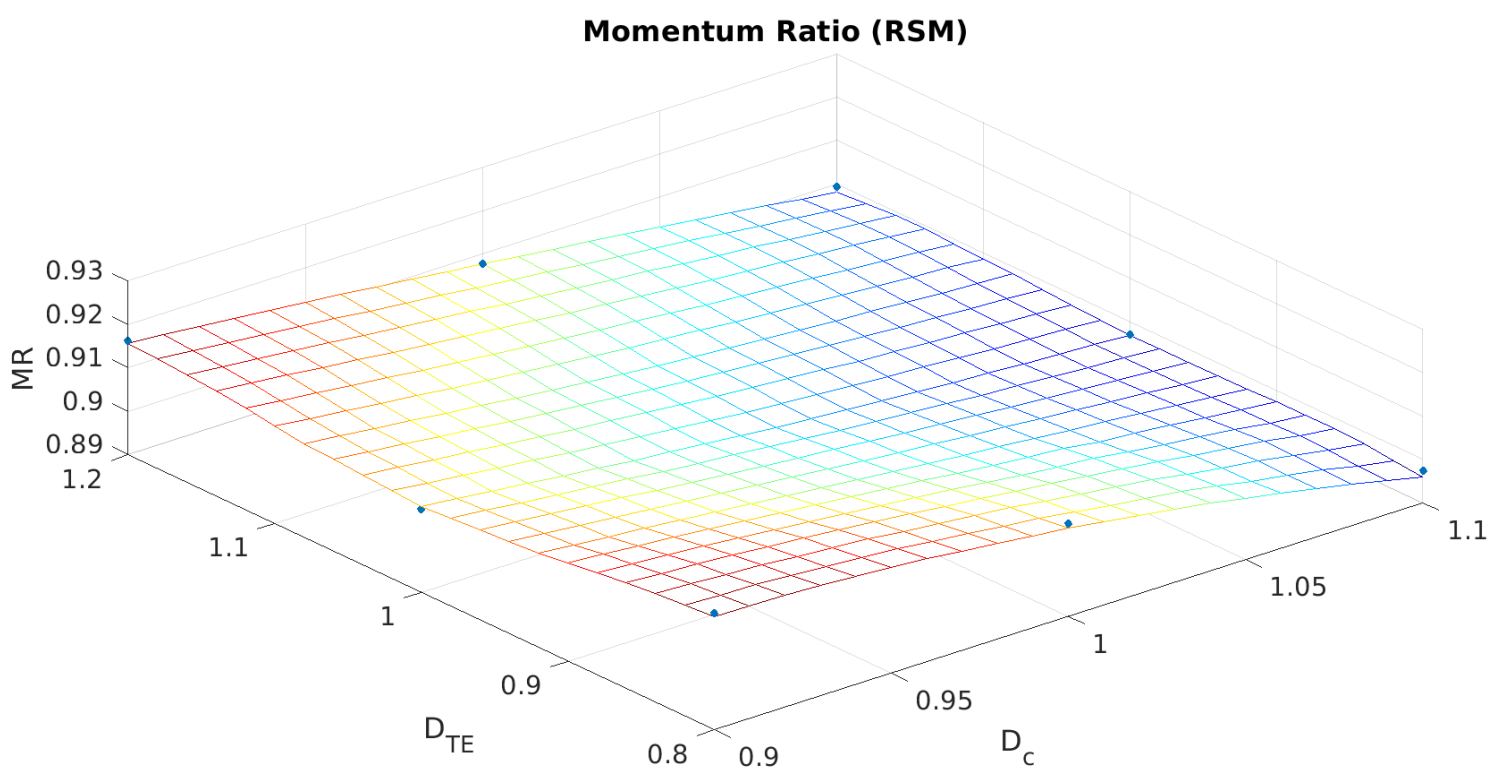

(b) Reynolds stress model

Figure 9. Momentum Ratio $M R$ (Padè polynomials).

Concerning the $B R$ RSs showed from Figures 12a to 13b, no remarkable differences are individuated by changing either the turbulence or the UQ methodology. The latter outcome is ascribable to the smooth distribution of the deterministic points. Looking at the RSs it is possible to note that $B R$ varies by a non-negligible factor (up to $20 \%$ as previously underlined) mainly depending on the $\mathrm{D}_{c}$ value. 


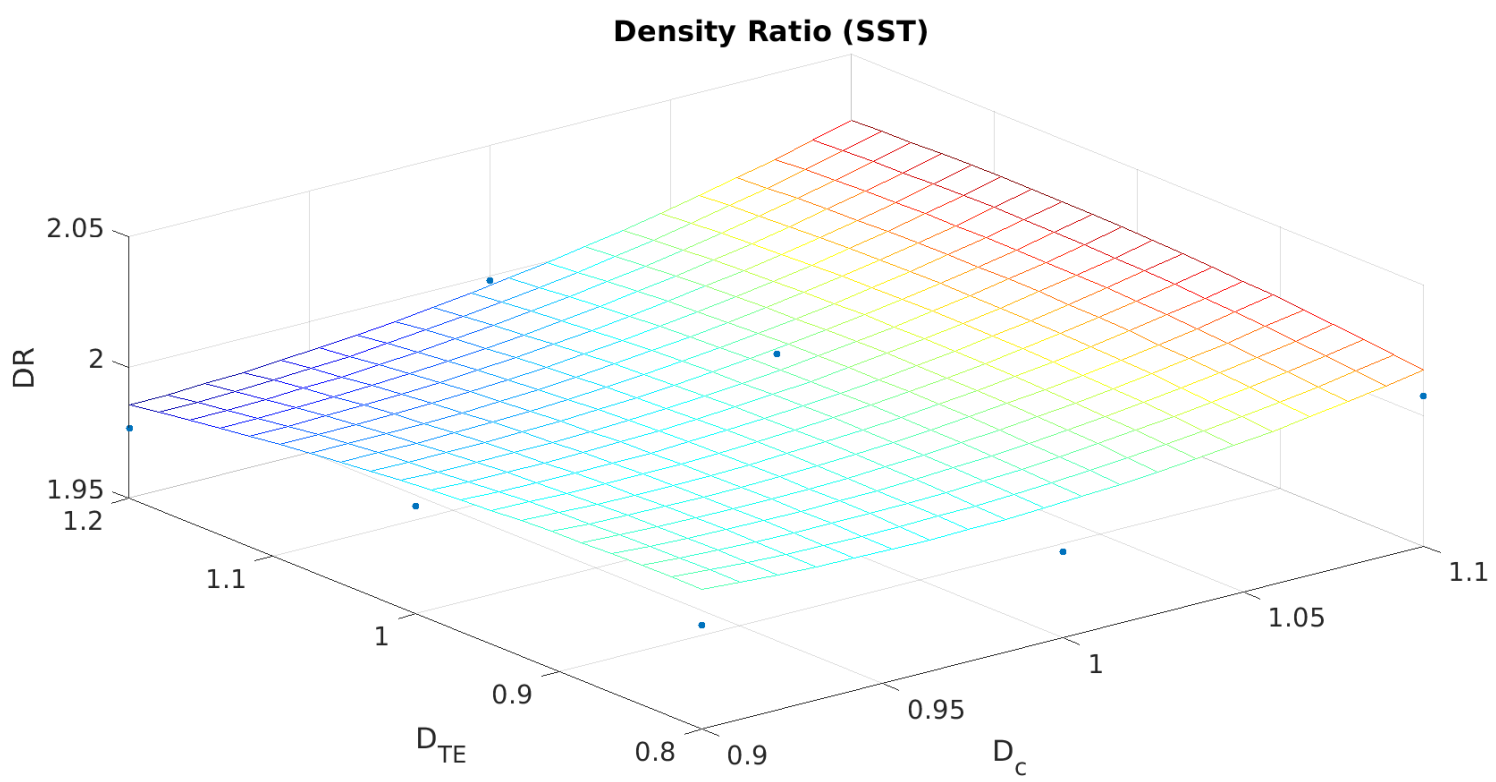

(a) SST model

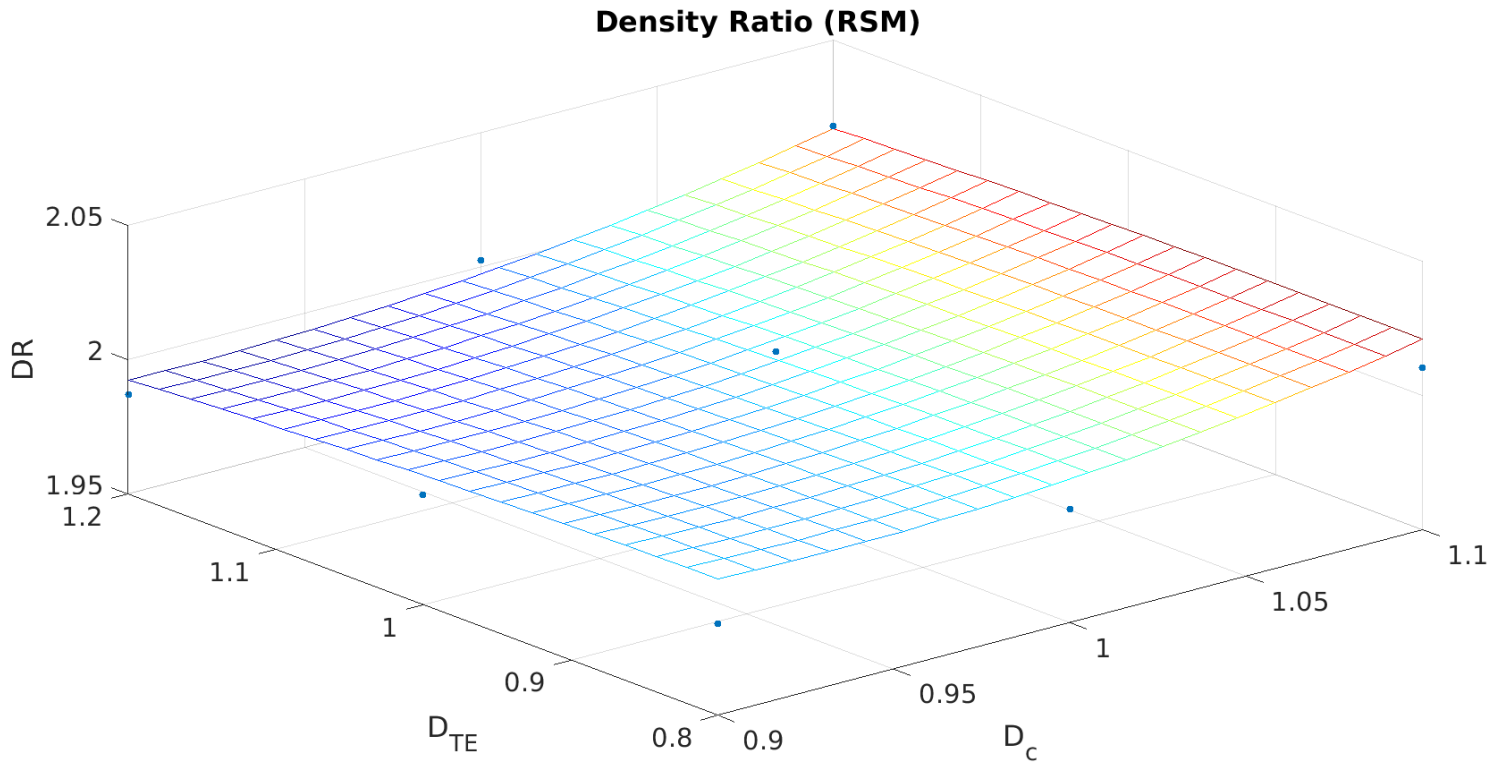

(b) Reynolds stress model

Figure 10. Density Ratio DR (Hermite polynomials).

Generally speaking, the impact of the variation of $D_{T E}$ on the selected parameters seems to be negligible for all the investigated cases. That outcome could be explained considering that the calculations have been performed with steady flow assumption, which neglects the possibility that a variation in the $D_{T E}$ value would affect the shedding frequency and the time-averaged shock intensity and impact location. However, more analyses are necessary on a symmetric control volume with periodic conditions to verify that guess. 


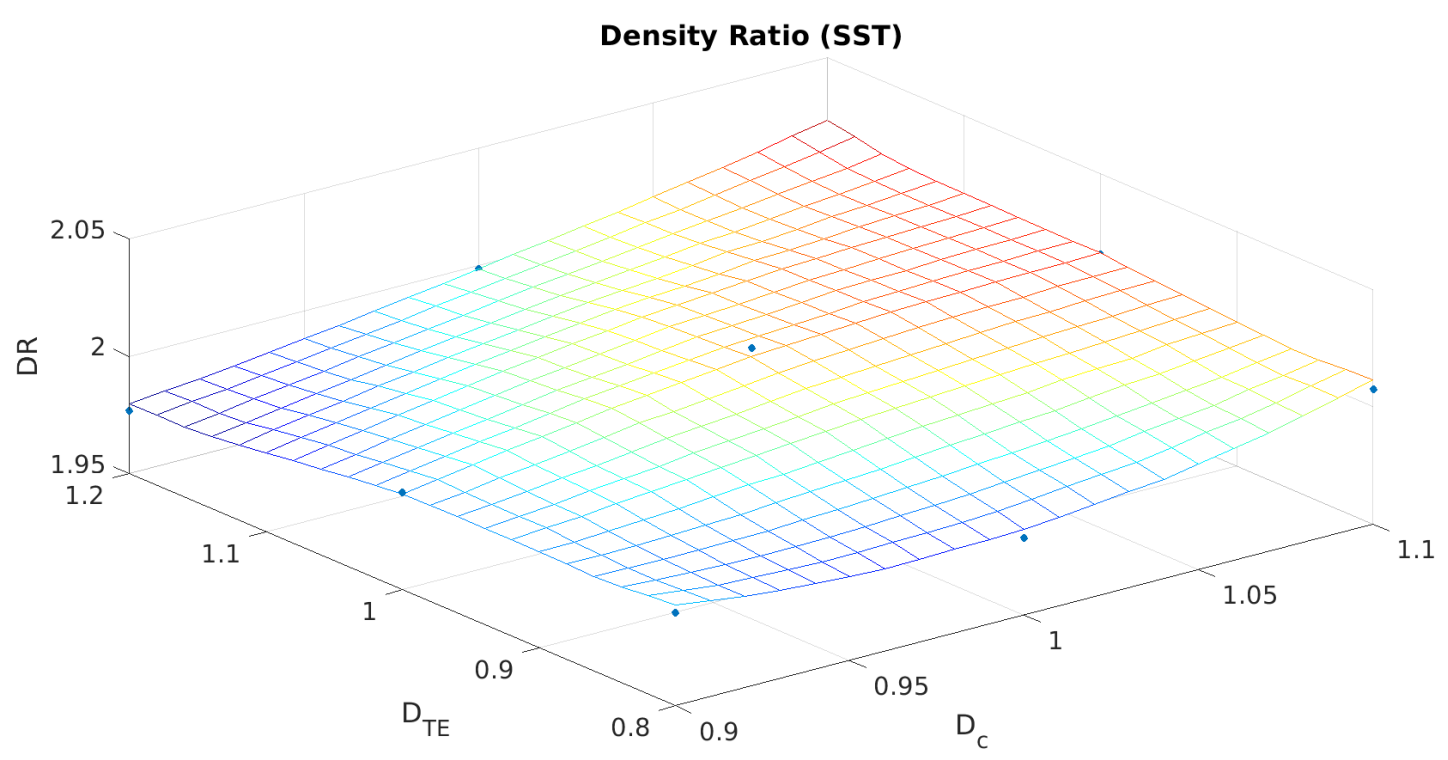

(a) SST model

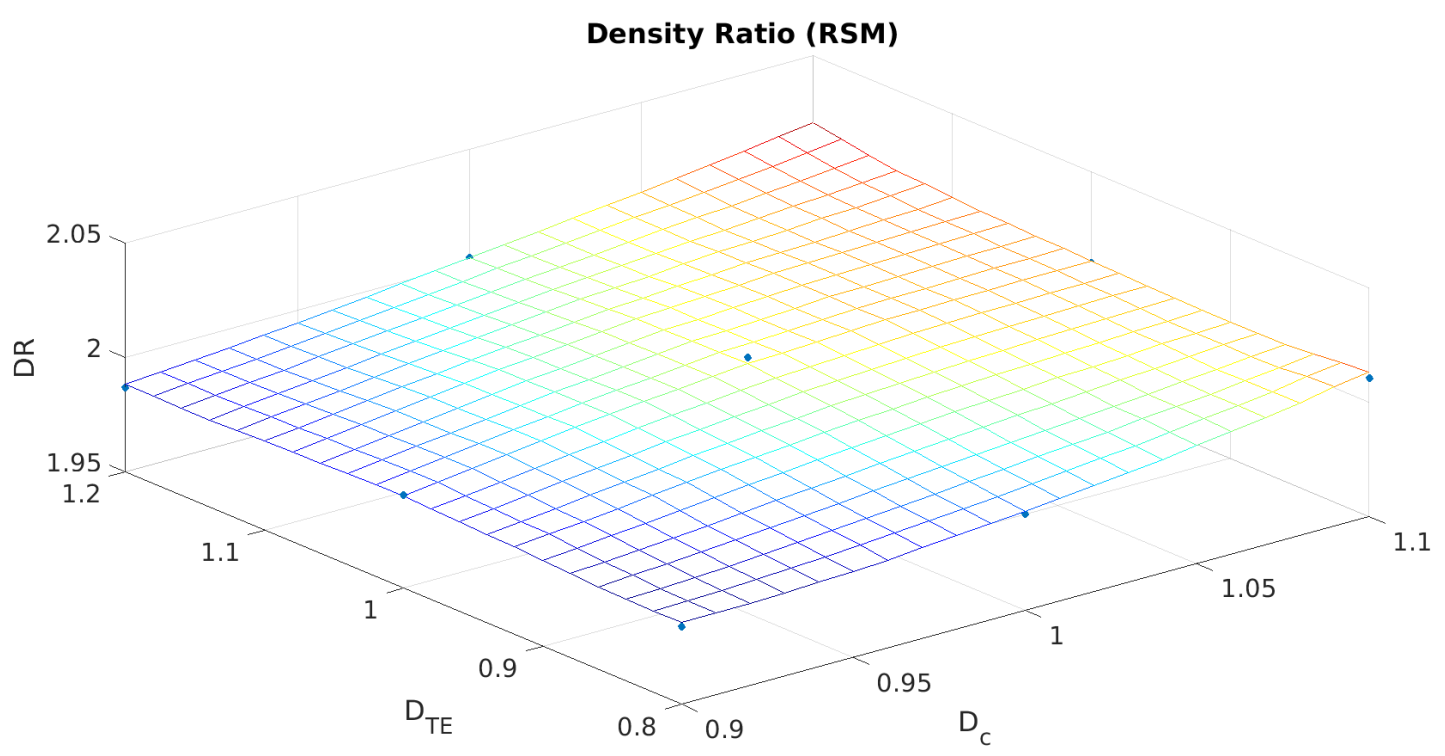

(b) Reynolds stress model

Figure 11. Density Ratio $D R$ (Padè polynomials).

From a technological point of view, it is of major interest to determine the mean value and the standard deviation (STD) associated with the statistical analysis. For that reason, the values obtained for the nominal case and using the two UQ methodologies are reported in Table 4. Values obtained using the UQ methodologies are quite close (within an accuracy of $1 \%$ ) to the deterministic values. What is primarily interesting are the associated STD values that represent the numerical uncertainty associated with manufacturing deviations. As can be observed, STD values associated with $D R, M R$ and $C_{D}$ are below $1.1 \%$. Concerning $B R$, STD values are between $11.5 \%$ and $13.5 \%$, thus demonstrating a non-negligible impact of manufacturing deviations on that fundamental parameters. It is worth underlining that the values obtained using Hermite polynomials and Padè-Legendre approximants are quite close with each other. It can be concluded that despite the higher ability in the reconstruction of RSs, to use Padè-Legendre approximants does not provide a relevant improvement in the evaluation of mean values and STDs (at least in a case where the RSs do not present steep curvature variations). 


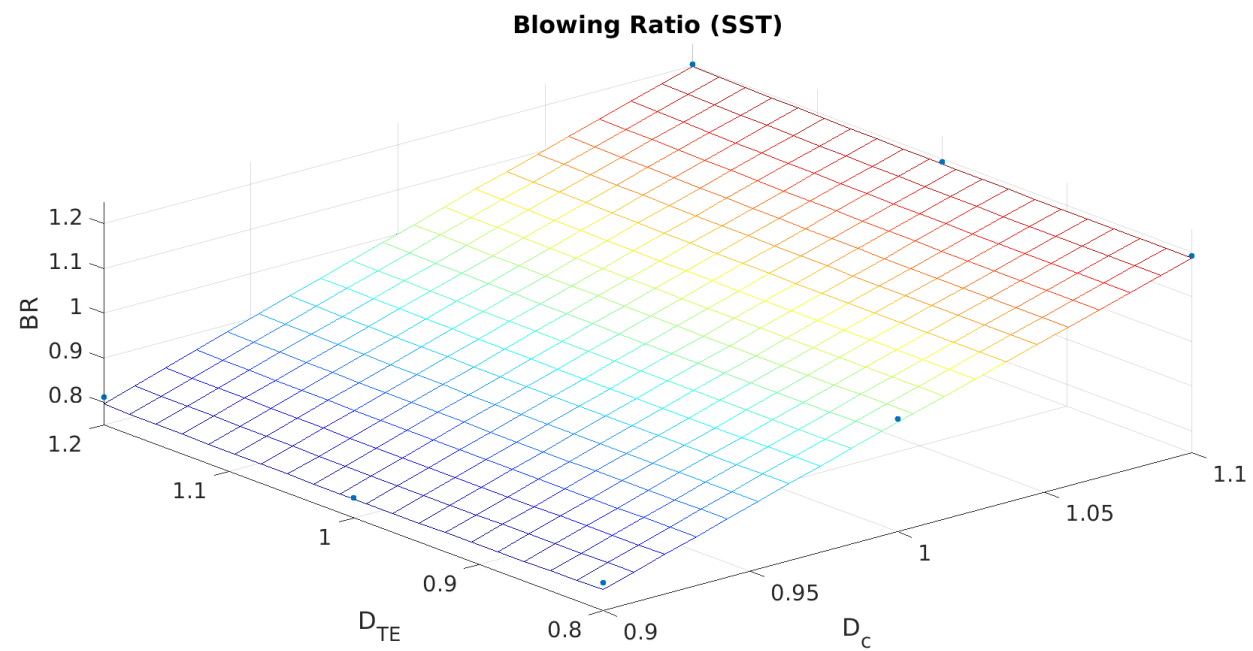

(a) SST model

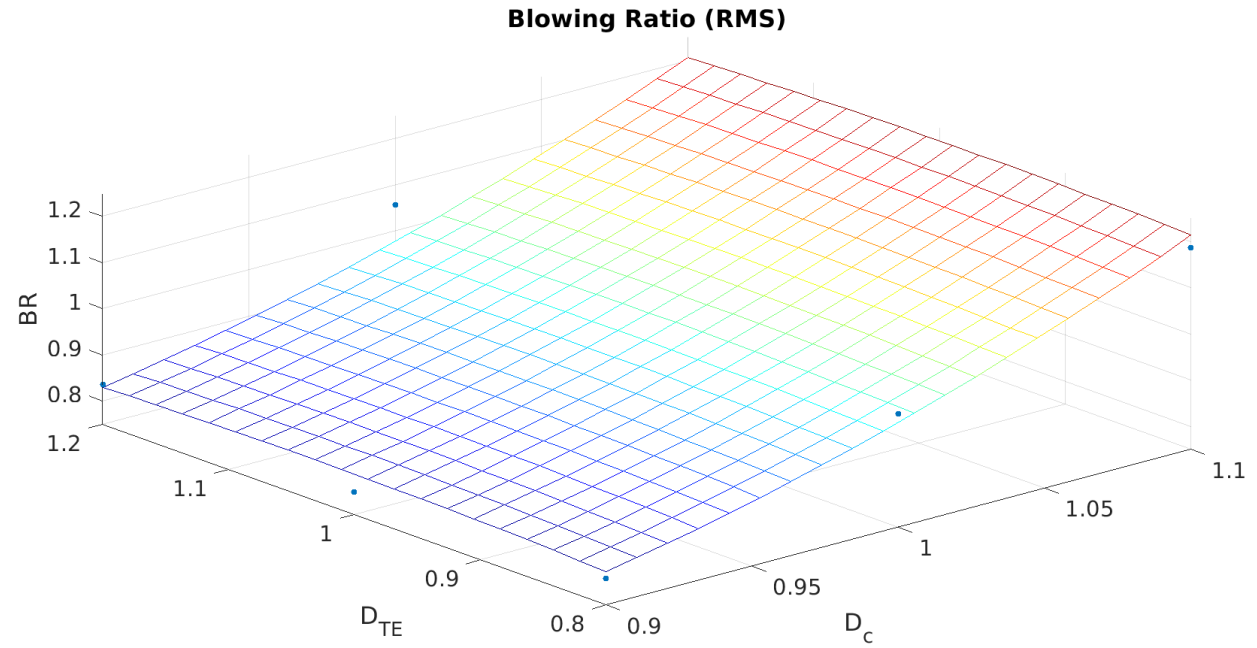

(b) Reynolds stress model

Figure 12. Blowing Ratio $B R$ (Hermite polynomials).

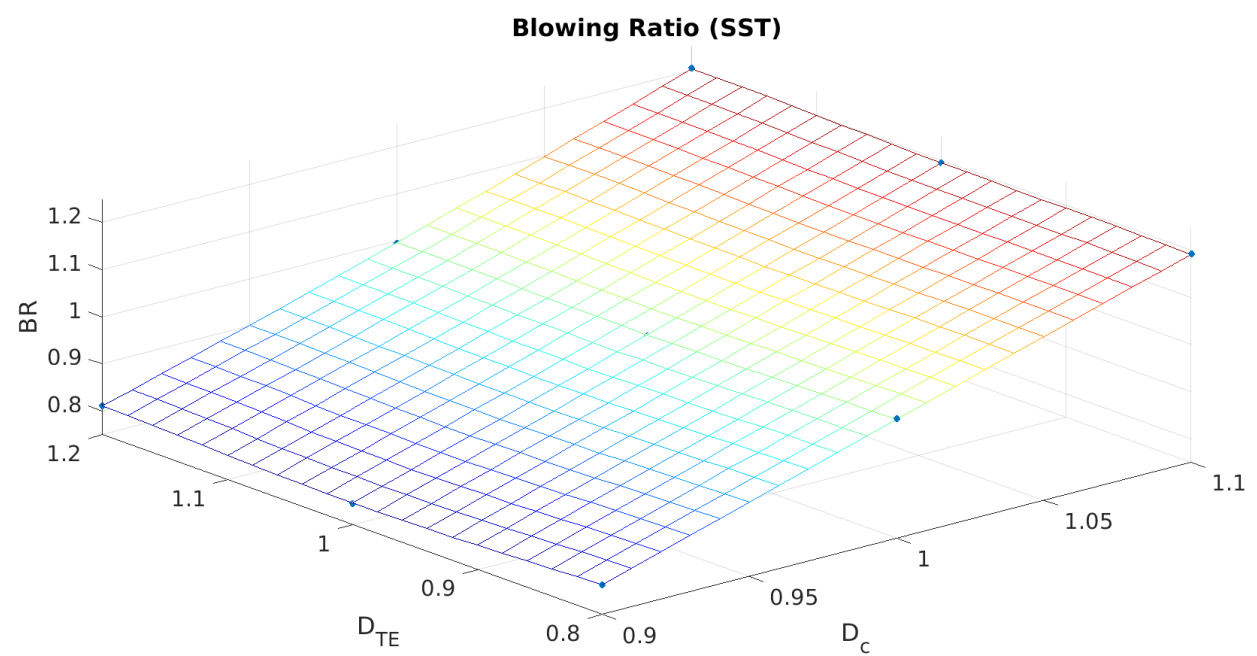

(a) SST Model

Figure 13. Cont. 


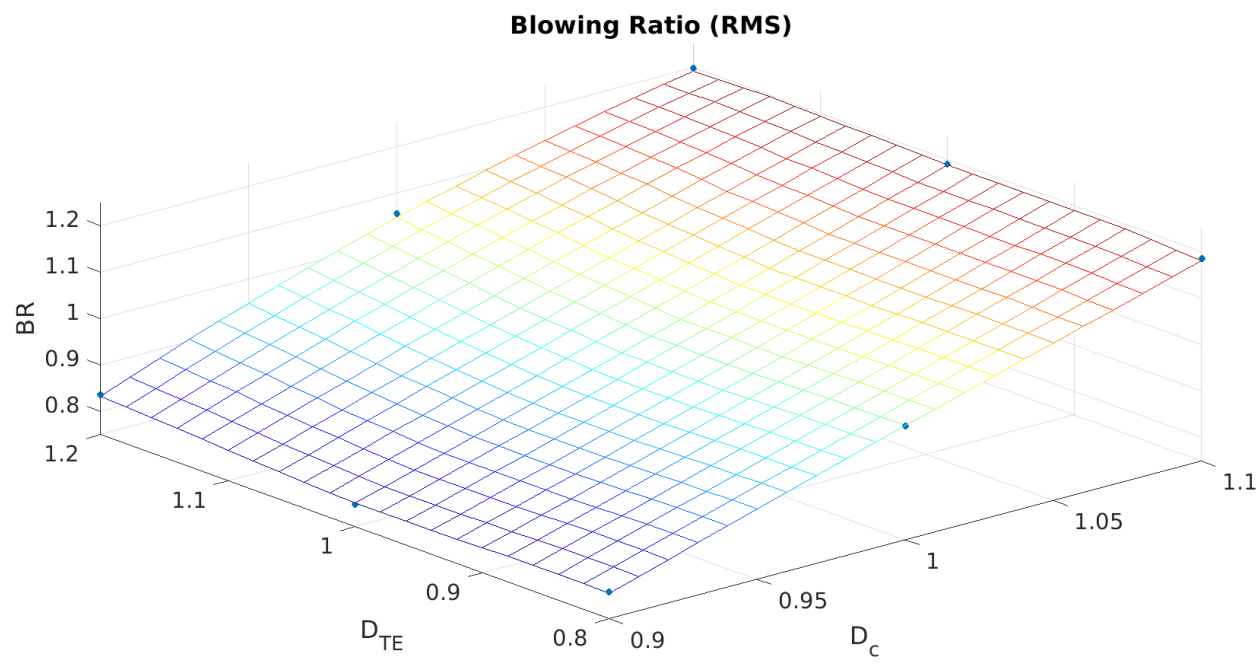

(b) Reynolds stress model

Figure 13. Blowing Ratio $B R$ (Padè polynomials).

Table 4. Mean value and standard deviation of $\mathrm{BR}, \mathrm{DR}, \mathrm{MR}$ e $C_{D}$ parameters and comparison with deterministic data.

\begin{tabular}{cccccc}
\hline$C_{D}[-]$ & Deterministic & $\begin{array}{c}\text { Mean Value } \\
\text { (Hermite) }\end{array}$ & $\begin{array}{c}\text { Standard Deviation } \\
\text { (Hermite) }\end{array}$ & $\begin{array}{c}\text { Mean Value } \\
\text { (Padè) }\end{array}$ & $\begin{array}{c}\text { Standard Deviation } \\
\text { (Padè) }\end{array}$ \\
\hline SST & 0.736 & 0.737 & 0.001 & 0.738 & 0.004 \\
RSM & 0.732 & 0.734 & 0.002 & 0.736 & 0.004 \\
\hline BR [-] & Deterministic & $\begin{array}{c}\text { Mean Value } \\
\text { (Hermite) }\end{array}$ & $\begin{array}{c}\text { Standard Deviation } \\
\text { (Hermite) }\end{array}$ & $\begin{array}{c}\text { Mean Value } \\
\text { (Padè) }\end{array}$ & $\begin{array}{c}\text { Standard Deviation } \\
\text { (Padè) }\end{array}$ \\
\hline SST & 0.990 & 0.994 & 0.115 & 0.999 & 0.135 \\
RSM & 0.985 & 0.999 & 0.125 & 1.005 & 0.131 \\
\hline DR [-] & Deterministic & Mean Value & Standard Deviation & $\begin{array}{c}\text { Mean Value } \\
\text { (Padè) }\end{array}$ & $\begin{array}{c}\text { Standard Deviation } \\
\text { (Padè) }\end{array}$ \\
\hline (HST & 2.015 & 2.005 & 0.011 & 2.001 & 0.011 \\
RSM & 2.020 & 2.004 & 0.009 & 2.001 & 0.009 \\
\hline MR [-] & Deterministic & Mean Value & Standard Deviation & $\begin{array}{c}\text { Mean Value } \\
\text { (Padè) }\end{array}$ & $\begin{array}{c}\text { Standard Deviation } \\
\text { (Padè) }\end{array}$ \\
\hline SST & 0.898 & 0.903 & 0.006 & 0.905 & 0.007 \\
RSM & 0.901 & 0.904 & 0.005 & 0.906 & 0.006 \\
\hline
\end{tabular}

\section{Conclusions}

The main topic of the present activity is the evaluation of the impact of manufacturing deviations on the performance parameters $\left(C_{D}, B R, D R\right.$ and $\left.M R\right)$ of a film cooling configuration in presence of oblique shocks. A total amount of 18 steady simulations are used to take into consideration the geometrical uncertainty associated with the cooling-hole diameter and the dimension of the central plate trailing edge. Two different turbulence closures (SST and RSM) are used to underline the impact of the anisotropy on the calculation of the $\eta_{\text {aw }}$ 2-D maps. Two different UQ methodologies based on Hermite polynomials and Padè-Legendre approximants are used to determine the most suitable approach.

Concerning the deterministic results obtained for the nominal configuration, it is observed that the coolant is mainly confined close to the centerline and its mixing with the main flow is underestimated if compared with the available experimental map. The use of the RSM approach for turbulence modelling does not provide significant improvements in the calculation of $\eta_{\text {aw }}$. Those outcomes are 
in line with computations by other authors [29] and are associated with the under-prediction of the vertical mixing in the wake of a jet for high $B R$ values. Also, the $C_{D}$ and $B R$ parameters are compared with the experimental values and are found to be within the experimental accuracy and in line with literature data.

Concerning the UQ analysis, it is observed that $C_{D}, D R$ and $M R$ are not almost unaffected by the selected manufacturing deviations while $B R$ greatly depends on $\mathrm{D}_{c}$. No relevant impact is found using either SST model or RSM in combination with Hermite polynomials and Padè-Legendre approximants. The latter can generate Reynolds stresses that match the deterministic points with greater accuracy with respect to Hermite polynomials, but it does not mean that relevant changes are found in the calculation of mean values and STD. In fact, all the data obtained using the UQ methodologies are close (within an accuracy of $1.1 \%$ ) to the corresponding deterministic ones. STD values for $B R$ are between $11.5 \%$ and $13.5 \%$, thus confirming a non-negligible impact of manufacturing deviations.

Author Contributions: Conceptualization, S.S., M.C. and F.M.; methodology, S.S. and M.C.; software, S.S. and M.C.; validation, S.S., M.C. and A.F.; formal analysis, S.S. and M.C.; investigation, S.S., M.C. and A.F.; resources, S.S., M.C. and F.M.; data curation, S.S., M.C., A.F.; writing—original draft preparation, S.S., M.C. and F.M.; writing-review and editing, S.S., M.C. and F.M.; visualization, S.S. and M.C.; supervision, F.M.; project administration, S.S. and F.M.; funding acquisition, M.C. and F.M.

Funding: This research received no external funding.

Acknowledgments: The authors want to thank sincerely Francesco Martelli (University of Florence), Achmed Schulz (Institut für Thermische Strömungsmaschinen), Marco Ochs and Giulio Cantini for without them, this paper would not have been possible. The authors acknowledge the European Commission for supporting the experimental activity in the frame of the TATEF2 (Turbine Aero-Thermal External Flows 2) FP6 project.

Conflicts of Interest: The authors declare no conflict of interest.

\section{Nomenclature}

$\begin{array}{ll}\text { Symbol } & \\ A & \text { area } \\ a & \text { coefficient of the generic expansion } \\ C & \text { coefficient } \\ D & \text { diameter, domain } \\ H & \text { Hermite polynomial } \\ M a & \text { Mach number } \\ P & \text { generic polynomial for a PCM reconstruction } \\ p & \text { pressure } \\ P r & \text { Prandtl number } \\ R & \text { gas constant } \\ r & \text { recovery factor } \\ T & \text { temperature } \\ u & \text { velocity } \\ x & \text { quadrature points, deterministic functions } \\ \text { Subscripts } & \\ 0 & \text { stagnation } \\ 1 & \text { inlet } \\ 2 & \text { outlet } \\ a & \text { adiabatic } \\ c & \text { coolant } \\ d & \text { discharge } \\ h o l e & \text { relative to the cooling hole } \\ i, j & \text { generic counter } \\ i s & \text { isentropic } \\ m, m a i n & \text { main flow } \\ n & \text { order of the polynomial quadrature }\end{array}$




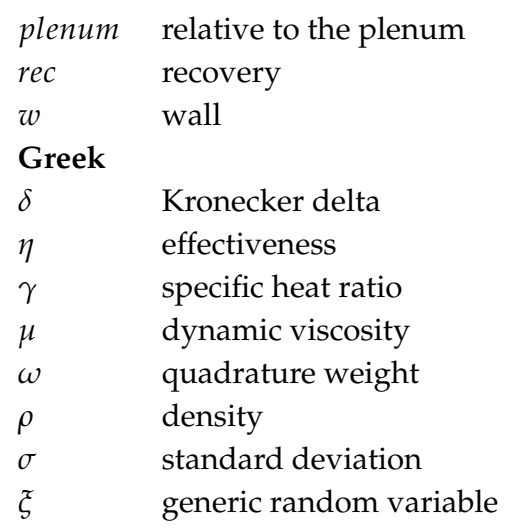

\section{Abbreviations}

1-D One-Dimensional

2-D Two-Dimensional

BR Blowing Ratio

CFD Computational Fluid Dynamics

DR Density Ratio

LES Large Eddy Simulation

MR Momentum Ratio

PC Polynomial Chaos

PCM Probabilistic Collocation Method

PDF Probability Distribution Function

PL Padè-Legendre

RANS Reynolds-Averaged Navier-Stokes

RS Response Surface

RSM Reynolds Stress Model

SRQ System Response Quantity

SST Shear Stress Transport

STD Standard Deviation

TE Trailing Edge

TET Turbine Entry Temperature

UQ Uncertainty Quantification

\section{References}

1. Salvadori, S.; Montomoli, F.; Martelli, F.; Adami, P.; Chana, K.; Castillon, L. Aerothermal study of the unsteady flow field in a transonic gas turbine with inlet temperature distortions. J. Turbomach. 2011, 133, 031030. [CrossRef]

2. Andreopoulos, J.; Rodi, W. Experimental investigation of jets in a crossflow. J. Fluid Mech. 1984, 138, 93-127. [CrossRef]

3. Leboeuf, F.; Sgarzi, O. The detailed structure and behavior of discrete cooling jets in a turbine. Ann. N. Y. Acad. Sci. 2001, 934, 95-109. [CrossRef] [PubMed]

4. Hagen, J.; Kurosaka, M. Corewise cross-flow transport in hairpin vortices-The "tornado effect". Phys. Fluids A Fluid Dyn. 1993, 5, 3167-3174. [CrossRef]

5. Chen, C.H.P.; Blackwelder, R.F. Large-scale motion in a turbulent boundary layer: A study using temperature contamination. J. Fluid Mech. 1978, 89, 1-31. [CrossRef]

6. Carnevale, M.; D'Ammaro, A.; Montomoli, F.; Salvadori, S. Film cooling and shock interaction: An uncertainty quantification analysis with transonic flows. In Proceedings of the ASME Turbo Expo 2014: Turbine Technical Conference and Exposition, Düsseldorf, Germany, 16-20 June 2014; p. V05BT13A001.

7. Montis, M.; Ciorciari, R.; Salvadori, S.; Carnevale, M.; Niehuis, R. Numerical prediction of cooling losses in a high-pressure gas turbine airfoil. Proc. Inst. Mech. Eng. Part A J. Power Energy 2014, 228, 903-923. [CrossRef] 
8. Ligrani, P.; Saumweber, C.; Schulz, A.; Wittig, S. Shock wave-film cooling interactions in transonic flows. In Proceedings of the ASME Turbo Expo 2001: Power for Land, Sea, and Air, New Orleans, LA, USA, 4-7 June 2001; p. V003T01A019.

9. Ochs, M.; Schulz, A.; Bauer, H.J. Investigation of the influence of trailing edge shock waves on film cooling performance of gas turbine airfoils. In Proceedings of the ASME Turbo Expo 2007: Power for Land, Sea, and Air, Montreal, QC, Canada, 14-17 May 2007; pp. 465-474.

10. Salvadori, S.; Montomoli, F.; Martelli, F. Film-cooling performance in supersonic flows: effect of shock impingement. Proc. Inst. Mech. Eng. Part A J. Power Energy 2013, 227, 295-305. [CrossRef]

11. Montomoli, F.; Amirante, D.; Hills, N.; Shahpar, S.; Massini, M. Uncertainty quantification, rare events, and mission optimization: Stochastic variations of metal temperature during a transient. J. Eng. Gas Turbines Power 2015, 137, 042101. [CrossRef]

12. El-Gabry, L.A.; Heidmann, J.D. Numerical study on the sensitivity of film cooling CFD results to experimental and numerical uncertainties. Int. J. Comput. Methods Eng. Sci. Mech. 2013, 14, 317-328. [CrossRef]

13. Montomoli, F.; Massini, M.; Salvadori, S.; Martelli, F. Geometrical uncertainty and film cooling: Fillet radii. J. Turbomach. 2012, 134, 011019. [CrossRef]

14. Carnevale, M.; Montomoli, F.; D'Ammaro, A.; Salvadori, S.; Martelli, F. Uncertainty quantification: A stochastic method for heat transfer prediction using LES. J. Turbomach. 2013, 135, 051021. [CrossRef]

15. Ochs, M.; Schulz, A.; Bauer, H.J. Design and commissioning of a transonic test section for the investigation of shock wave-film cooling interactions. In Proceedings of the ASME Turbo Expo 2006: Power for Land, Sea, and Air, Barcelona, Spain, 8-11 May 2006; pp. 619-628.

16. Menter, F. Zonal two equation kw turbulence models for aerodynamic flows. In Proceedings of the 23rd Fluid Dynamics, Plasmadynamics, and Lasers Conference, Orlando, FL, USA, 6-9 July 1993; p. 2906.

17. Hanjalić, K.; Launder, B. A Reynolds stress model of turbulence and its application to thin shear flows. J. Fluid Mech. 1972, 52, 609-638. [CrossRef]

18. Bontempo, R.; Manna, M. Highly accurate error estimate of the momentum theory as applied to wind turbines. Wind Energy 2017, 20, 1405-1419. [CrossRef]

19. Andreini, A.; Bacci, T.; Insinna, M.; Mazzei, L.; Salvadori, S. Hybrid RANS-LES modeling of the aerothermal field in an annular hot streak generator for the study of combustor-turbine interaction. J. Eng. Gas Turbines Power 2017, 139, 021508. [CrossRef]

20. Wilcox, D.A. Simulation of transition with a two-equation turbulence model. AIAA J. 1994, 32, $247-255$. [CrossRef]

21. Salvadori, S.; Carnevale, M.; Ahlfeld, R.; Montomoli, F.; Martelli, F. Stochastic variation of the aero-thermal flow field in a cooled high-pressure transonic vane configuration. In Proceedings of the 12th European Conference on Turbomachinery Fluid Dynamics and Thermodynamics, ETC 2017, Stockholm, Sweden, 3-7 April 2017.

22. Eckert, E.R.G.; Drake, R.M., Jr. Analysis of Heat and Mass Transfer; Hemisphere Publishing: New York, NY, USA, 1987.

23. Garg, V.K.; Rigby, D.L. Heat transfer on a film-cooled blade-effect of hole physics. Int. J. Heat Fluid Flow 1999, 20, 10-25. [CrossRef]

24. Adami, P.; Martelli, F.; Montomoli, F.; Saumweber, C. Numerical investigation of internal crossflow film cooling. In Proceedings of the ASME Turbo Expo 2002: Power for Land, Sea, and Air, Amsterdam, The Netherlands, 3-6 June 2002, pp. 51-63.

25. Acharya, S.; Leedom, D.H. Large eddy simulations of discrete hole film cooling with plenum inflow orientation effects. J. Heat Transf. 2013, 135, 011010. [CrossRef]

26. Li, H.; Ghaly, W.; Hassan, I. The formation of counter-rotating vortex pair and the nature of liftoff-reattachment in film-cooling flow. Fluids 2016, 1, 39. [CrossRef]

27. Kodzwa, P.M. Measurements of Film Cooling Performance in a Transonic Single Passage model. Ph.D. Thesis, Stanford University, Stanford, CA, USA, 2005.

28. Ochs, M.; Schulz, A.; Bauer, H.J. Report on Tests with Steady Shocks; Technical Report; Universitt Karlsruhe, Institut fuer Thermische Stroemungsmaschinen: Karlsruhe, Germany, 2007.

29. El-Gabry, L.; Heidmann, J.; Ameri, A. Numerical Analysis of Film Cooling at High Blowing Ratio; NASA Glenn Research Center: Cleveland, OH, USA, 2009. 
30. Ahlfeld, R.; Montomoli, F.; Carnevale, M.; Salvadori, S. Autonomous uncertainty quantification for discontinuous models using multivariate Padé approximations. J. Turbomach. 2018, 140, 041004. [CrossRef]

31. Gritsch, M.; Schulz, A.; Wittig, S. Discharge coefficient measurements of film-cooling holes with expanded exits. In Proceedings of the ASME 1997 International Gas Turbine and Aeroengine Congress and Exhibition, Orlando, FL, USA, 2-5 June 1997; p. V003T09A030.

32. Saracoglu, B.; Paniagua, G.; Salvadori, S.; Tomasoni, F.; Duni, S.; Yasa, T.; Miranda, A. Trailing edge shock modulation by pulsating coolant ejection. Appl. Therm. Eng. 2012, 48, 1-10. [CrossRef]

33. Logan, E., Jr. Handbook of Turbomachinery; CRC Press: Boca Raton, FL, USA, 2003.

34. Griffini, D.; Salvadori, S.; Carnevale, M.; Montomoli, F. Uncertainty Quantification in Hydrodynamic Bearings. Energy Procedia 2018, 148, 884-891. [CrossRef]

35. Wiener, N. The homogeneous chaos. Am. J. Math. 1938, 60, 897-936. [CrossRef]

36. Xiu, D.; Karniadakis, G.E. The Wiener-Askey polynomial chaos for stochastic differential equations. SIAM J. Sci. Comput. 2002, 24, 619-644. [CrossRef]

37. Montomoli, F.; Carnevale, M.; D’Ammaro, A.; Massini, M.; Salvadori, S. Uncertainty Quantification in Computational Fluid Dynamics and Aircraft Engines; Springer: Berlin, Germany, 2015.

38. Chantrasmi, T.; Doostan, A.; Iaccarino, G. Padé-Legendre approximants for uncertainty analysis with discontinuous response surfaces. J. Comput. Phys. 2009, 228, 7159-7180. [CrossRef]

39. Hesthaven, J.S.; Kaber, S.M.; Lurati, L. Padé-Legendre interpolants for Gibbs reconstruction. J. Sci. Comput. 2006, 28, 337-359. [CrossRef]

40. Abramowitz, M.; Stegun, I.A. Handbook of Mathematical Functions: With Formulas, Graphs, and Mathematical Tables; Courier Corporation: North Chelmsford, MA, USA, 1965; Volume 55.

(C) 2019 by the authors. Licensee MDPI, Basel, Switzerland. This article is an open access article distributed under the terms and conditions of the Creative Commons Attribution (CC BY) license (http:/ / creativecommons.org/licenses/by/4.0/). 